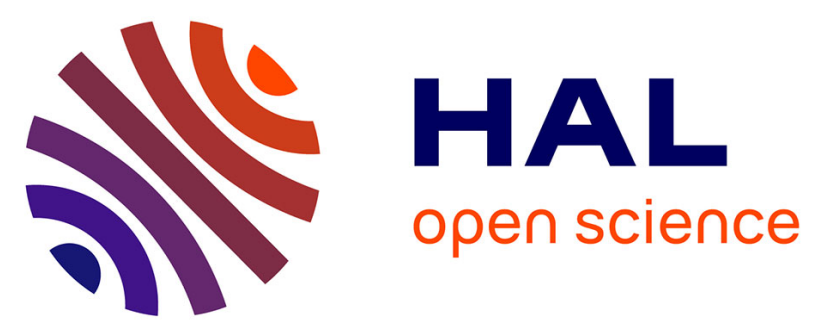

\title{
A Simplified Electrochemical and Thermal Aging Model of LiFePO4-Graphite Li-ion Batteries: Power and Capacity Fade Simulations
}

Eric Prada, D. Di Domenico, Y. Creff, J. Bernard, Valérie Sauvant-Moynot, François Huet

\section{To cite this version:}

Eric Prada, D. Di Domenico, Y. Creff, J. Bernard, Valérie Sauvant-Moynot, et al.. A Simplified Electrochemical and Thermal Aging Model of LiFePO4-Graphite Li-ion Batteries: Power and Capacity Fade Simulations. Journal of The Electrochemical Society, 2013, 160 (4), pp.A616-A628. $10.1149 / 2.053304$ jes . hal-00788280

\section{HAL Id: hal-00788280 \\ https://hal.sorbonne-universite.fr/hal-00788280}

Submitted on 14 Feb 2013

HAL is a multi-disciplinary open access archive for the deposit and dissemination of scientific research documents, whether they are published or not. The documents may come from teaching and research institutions in France or abroad, or from public or private research centers.
L'archive ouverte pluridisciplinaire HAL, est destinée au dépôt et à la diffusion de documents scientifiques de niveau recherche, publiés ou non, émanant des établissements d'enseignement et de recherche français ou étrangers, des laboratoires publics ou privés. 


\title{
A Simplified Electrochemical and Thermal Aging Model of $\mathrm{LiFePO}_{4}$-Graphite Li-ion Batteries: Power and Capacity Fade Simulations
}

\author{
E. Prada ${ }^{a, z}$, D. Di Domenico ${ }^{a}$, Y. Creff ${ }^{a}$, J. Bernard $^{\text {a }}$, V.Sauvant-Moynot ${ }^{\text {a }}$ F. Huet $^{\text {b }}$ \\ ${ }^{a}$ IFP Energies Nouvelles, Rond-Point de l'échangeur de Solaize, Solaize, France \\ ${ }^{\mathrm{b} U P R} 15$ - LISE; CNRS; UPMC Univ Paris 06, F-75005 Paris, France
}

\begin{abstract}
In this paper, an isothermal physics-based aging model from the literature is modified and extended to simulate both capacity and power fade of a commercial $\mathrm{LiFePO}_{4}$-graphite Li-ion battery. Compared to the isothermal reference, the mechanism of porosity modification due to the Solid Electrolyte Interphase (SEI) film growth at the negative electrode is integrated in the present electrochemical and thermal model to establish theoretical correlations between capacity and power fade of the system. The aging model includes different contributions of the cell impedance increase such as the SEI film resistance and the electrolyte mass transport resistance due to the mitigation of the negative electrode porosity. Experimental databases from literature and specific experiments coupling endurance tests and Electrochemical Impedance Spectroscopy results, are used to calibrate and validate the correlated power and capacity loss simulations for both calendar and classic galvanostatic cycling operating conditions. The analysis of the experimental data points out that an additional possible aging mechanism such as cracking and fracture of the SEI layer could play an important role for cycling operating conditions and accelerate the electrochemical mechanisms. The impact of physical and design parameters on the power and capacity theoretical correlations are discussed. The limits of applicability of the present model are also discussed in this paper.

${ }^{\mathrm{z}}$ E-mail: eric.prada@ifpen.fr
\end{abstract}


Due to their high power and energy densities, Li-ion technologies are the leading battery systems for the new generations of electric vehicles (EVs) and plug-in hybrid electric vehicles (PHEVs). These batteries can suffer from aging issues during service leading to energy and power losses. Aging of Li-ion cells has been widely studied to understand the underlying mechanisms, improve materials and management, and decrease the aging kinetics. To get insight into these performance degradations, research efforts have been dedicated to model Li-ion cell aging, namely capacity loss or impedance increase, and quantify the impact of aging factors. ${ }^{1,2}$ In spite of intensive investigations on various positive and negative electrode chemistries, these aging phenomena are not always well understood nor quantified and the combined impacts of temperature (T), depth of discharge (DOD), and current intensity (I) still remain difficult to quantify and manage. Even if most aging mechanisms of Li-ion cells have been experimentally identified and described in literature, these phenomena are complex, chemistry dependent and can interact between each other and give different evolution shapes of capacity loss and power fade. From low temperature where Li plating can occur, ${ }^{3,4}$ to elevated temperature where loss of active material can be preponderant, ${ }^{5}$ the solid electrolyte interphase (SEI) growth at the negative electrode/electrolyte interface is one of the most studied mechanism until now. In most aging models, this mechanism leads to quadratic evolution shapes of the capacity loss and impedance rise in the first phases of the battery life, as described by Spotnitz. ${ }^{1}$

In order to model the SEI layer growth, the approaches encountered in literature are either semi-empirical or physics-based. Semi-empirical approaches have been widely described. ${ }^{1,6-8}$ In particular, Gerschler et al. ${ }^{8}$ developed a power-law aging model integrating the dynamics of SEI growth and distinguishing calendar and cycling effects. This model was integrated into a spatially resolved dynamic impedance-based model to take into account thermal gradients inside the cell. Based on a grey-box approach, this modelling technique is 
rather simple to calibrate but it failed in accurately describing the relationship between capacity and power loss. Moreover, the availability of these semi-empirical approaches is strictly limited to the range of calibration of the model. On the other hand, the electrochemical models, proposed for the first time by Newman and Thomas-Alyea, ${ }^{9}$ allow description of the aging reactions occurring at the electrodes interfaces on a physical and chemical basis. This latter modeling approach is more adapted to describe and quantify aging mechanisms at the electrode level, since it integrates the variability of the operating conditions. For simulation purposes, phenomenological Single-Particle (SP) models are preferred to Pseudo-TwoDimensional (P2D) model ${ }^{10}$ to save computing resources. As detailed in the following paragraphs, many authors have used physics-based approaches to study Li-ion cell aging with a specific focus on two main phenomena, namely the mechanism of SEI growth and the evolution of design parameters such as the negative electrode porosity.

Even if largely reported, the physical and chemical mechanisms of SEI growth are not universally adopted. The SEI film on the negative electrode material is believed to be composed of two distinct layers. As described by Christensen and Newman, ${ }^{11}$ the inner layer, which is made of inorganic products $\left(\mathrm{Li}_{2} \mathrm{CO}_{3}, \mathrm{LiF}, \mathrm{Li}_{2} \mathrm{O}, \mathrm{LiCl}\right)$ is around 1.5 to $2 \mathrm{~nm}$ thick, whereas the porous outer layer which is composed of reduced organic products $\left(\left(\mathrm{CH}_{2} \mathrm{OCO}_{2} \mathrm{Li}\right)_{2},\left(\mathrm{CH}_{2} \mathrm{CH}_{2} \mathrm{OCO}_{2} \mathrm{Li}\right)_{2} \ldots\right)$, can be on the order of $100 \mathrm{~nm}$ thick. In order to investigate the SEI growth, these authors developed a continuum-scale mathematical model to describe the transport of $\mathrm{Li}$ and electrons through the SEI. Recently, Colclasure et al. developed a SEI growth model based on a SP approach and incorporating chemical kinetics and multicomponent species transport. ${ }^{12}$ Considering the SEI as a non-porous medium, their model is coupled at its boundaries with an intercalation process at the SEI/particle interface and a Li-ion transport process at the SEI/electrolyte interface. 
Concerning capacity fade, Danilov and Notten ${ }^{13}$ used the hypothesis of electron tunnelling through the SEI to describe the capacity fade of $\mathrm{LiCoO}_{2}-\mathrm{C}$ technology. Ploehn et al. ${ }^{14}$ used the hypothesis of solvent diffusion through the SEI but without considering the side reaction kinetics. Safari et al. ${ }^{2}$ extended the previous model by incorporating the kinetics of solvent reduction reaction at the surface of the carbon material.

The modification of the negative electrode porosity due to SEI growth, was theoretically modelled by Sikha et al. ${ }^{15}$ More recently, Jannesari et al. ${ }^{16}$ modeled the variation of SEI thickness in the depth of the negative electrode of a Li-ion battery. This group demonstrated by simulations that improving the ionic conductivity significantly prolongs the lifetime of the battery owing to a uniform side reaction along the electrode.

In all the abovementioned modeling studies, the authors focused on either capacity fade or impedance rise. Capacity fade is generally attributed to irreversible consumption of Li ions, whereas impedance rise is related to the growing SEI layer resistivity and sometimes to the evolutions of electrolyte mass transport properties within the cell. To the best of our knowledge, no theoretical correlations between capacity fade and power loss have been clearly reported and validated on extensive experimental data. In this work, the model of SEI growth by solvent diffusion developed in Ref. 2 is adapted and improved by introducing the dynamic evolution of the negative electrode porosity in a lumped parameter approach. The modelling objective described in this paper is to design a simple physics-based model that could be easily calibrated and used for battery-management-system (BMS) applications, rather than a complex SEI formation model, as described in Ref. 11 with detailed mechanisms for ionic and electronic transport in the film.

After the description of the electrochemical and thermal nominal model developed recently, ${ }^{17}$ the theoretical equations of capacity and power losses of the aging model are detailed. In the next section, the experimental calibration and validation of the model are 
presented for both calendar and cycling operating conditions. The theoretical correlations between capacity fade and power loss are experimentally confirmed through Electrochemical Impedance Spectroscopy (EIS) experiments coupled with endurance tests. The theoretical limitations of the present model are assessed by analyzing the differences between simulations and experimental data for cycling operations and potential improvements of the present model are suggested. Finally, the impact of physical and design parameters on the theoretical correlations between power and capacity losses are discussed.

\section{Electrochemical and thermal aging model development}

Nominal electrochemical and thermal battery model.- A SP electrochemical and thermal model was developed in a previous paper from the Pseudo-2D mathematical structure to calculate the electrical charge delivered by a Li-ion cell at different temperatures and constant current regimes in both charge and discharge operations. ${ }^{17}$ The schematic representation of the 1D ( $z$ direction) model of the battery cell of thickness $L$ (Fig. 1 in Ref. 17 ) is composed of three solid porous media, namely the positive electrode (thickness $\delta_{p}$ and porosity $\varepsilon_{e, p}$ ), the separator (thickness $\delta_{\text {sep }}$ and porosity $\varepsilon_{e, s e p}$ ) and the negative electrode (thickness $\delta_{n}$ and porosity $\varepsilon_{e, n}$ ), wetted by a liquid electrolyte phase ensuring the transport of Li ions. In this model, the radius of the active material particles was current-dependent to improve the model predictions under fast charging/discharging operating conditions. It was then difficult to address dynamic profiles because of the current-sign change during the cycle so that the model was applied to continuous galvanostatic regimes only. In the present paper, for sake of simplicity the particle radius of the active material is not current-dependent, as traditionally performed in reported battery models.

The main electrochemical equations of the SP model are detailed in Table I. Considering spherical particles at the negative and positive electrodes, the concentration, $c_{s}(r)$, of $\mathrm{Li}^{+}$species in the solid phase is classically governed by Eq. 1 while the $\mathrm{Li}^{+}$ 
concentration, $c_{e}$, in the electrolyte phase at any time along the negative electrode / separator / positive electrode cell of thickness $L$ is given by Eq. 2. Adopting a lumped parameter approach, the overpotentials, $\bar{\eta}$, of both electrodes were expressed as the sum of the kinetic, $\bar{\eta}^{k}$, and diffusion, $\bar{\eta}^{\text {diff }}$, overpotentials (Eq. 4). Assuming a symmetry in the charge transfer coefficients $\left(\alpha_{\mathrm{ox}}=\alpha_{\mathrm{red}}=\alpha=0.5\right)$, the Butler-Volmer relations (Eq. 3) giving the Faradaic current density per unit volume, $j_{f}$, can be inverted to express the kinetic overpotential as a function of $\xi=\bar{j}_{f} /\left(2 a_{s} i_{0}\right)$ (Eq. 5). For Li-ion batteries, the symmetry hypothesis is assumed in control-oriented physics-based battery models to break the algebraic equations and to simplify the model structure, especially for BMS purposes. In practice, this means that the charge transfer is kinetically similar in charge and discharge, which seems to be a reasonable assumption. The diffusion overpotentials in the solid phase, $\bar{\eta}^{\text {diff }}$, have been expressed as the difference between the thermodynamic equilibrium potentials, $U$, taken at the surface and at the centre of the spherical particles (Eq. 6). In this equation, $\theta^{s}=c_{s}^{s} / c_{s, \max }$ and $\theta^{b}=c_{s}^{b} / c_{s, \text { max }}$ denote the normalized inserted Li ion concentration at the surface and in the bulk of the electrode. The mass-transport overpotential in the electrolyte, $\bar{\eta}_{e}^{m t}$, is expressed as the difference between the potentials of the electrolytic phase taken at the extremities of the electrodes (Eq. 7). Contrary to classical SP approaches, the mass-transport overpotential, $\bar{\eta}_{e}^{m t}$, is simply added in the voltage calculation. Mass transport is not coupled with the intercalation kinetics as it is generally done in higher-order electrochemical models, so that the model presented in Ref. 17 consists simply in a superposition of overpotentials due to kinetic and diffusion phenomena. Thus, in Eq. 8, the cell voltage can be expressed as a function of the design parameters.

As explained in Ref. 17, the temperature evolution and the thermal coupling with the electrochemical kinetics are governed by the heat transfer and energy balance equations given 
in Table II. This set of equations is the mathematical framework in which the physics-based aging equations will be integrated, as detailed in the next section.

Development of the physics-based electrochemical and thermal aging model.-Based on the hypothesis that the only source of aging is the solvent reduction leading to the SEI layer growth in the $\mathrm{LiFePO}_{4}$-graphite system investigated, the model developed by Safari et al. ${ }^{2}$ was modified and integrated into the electrochemical and thermal model presented in the previous section. No modification of the positive $\mathrm{LiFePO}_{4}$ electrode is assumed on the basis of its stability reported in the literature. ${ }^{18}$ In this work, no active material loss is considered and the loss of cyclable Li-ions is the main capacity loss mechanism, as reported in Refs. 18 and 19. Despite the fact that the SEI layer consists of an inner thin compact inorganic layer and an outer porous organic-containing layer, and because of the small thickness of the inner layer, the SEI film is described in the following developments as a single layer, the outer layer (Fig. 1).

The main hypotheses assumed in Ref. 2 are summarized in this section. Different reaction schemes have been proposed for the solvent (S) reduction but, for sake of simplicity, the following reaction is considered:

$$
S+2 e^{-}+2 L i^{+} \rightarrow P
$$

The solvent reduction is assumed to occur at the interface of the inner compact inorganic layer and the organic-containing outer layer. Since the potential window of interest is far from the equilibrium potential of the solvent reduction, $U_{s}$, it is reasonable to assume a Tafel behavior for the kinetics of the solvent reduction reaction (Eq. 15). In this equation, $i_{t}$ is the total current density, $i_{s}$ is the current density of the side reaction, $\phi_{s, n}$ is the electric potential of the negative electrode, $k_{f}$ and $\beta$ are the rate constant and charge-transfer coefficient of the side reaction, and $c_{\text {solv }}^{*}$ is the concentration of solvent molecules at the 
reactive interface in the SEI film of thickness $\delta_{S E I}$ and conductivity $\kappa_{S E I}$. The total current density is the sum of the parasitic current density of the side reaction and the intercalation current intensity defined as $i_{i n t}=\bar{j}_{f, n} / a_{s, n}$ (Eq. 16).

The transport of solvent molecules inside the SEI outer layer occurs via diffusion and convection, as represented by Eq. 17 under the assumption of a planar geometry of the SEI because of its small thickness. Convection is integrated in the transport equation to represent the apparent velocity of the solvent molecules that move in the opposite direction of the layer growth. In this equation, $c_{\text {solv }}$ and $D_{\text {solv }}$ are the concentration and diffusion coefficient of the solvent molecules in the SEI film. The solvent concentration is considered to be constant outside the SEI layer, as indicated in Fig. 1 and in the second boundary condition in Table III. This condition introduces a partition coefficient $\varepsilon_{S E I}$ under the assumption of a liquid medium in the SEI outer layer despite the fact that liquid and solid phases are actually present. In a sense, $\varepsilon_{S E I}$ can be considered as the volume fraction of electrolyte in the SEI porosity. As explained in Ref. 2, $\varepsilon_{S E I}$ is a key factor in the quality of the film and controls the rate of capacity fade of the battery. The evolution of the SEI thickness is given by Faraday's law (Eq. 18), where $M_{S E I}$ and $\rho_{S E I}$ are the molar mass and density of the film, respectively.

In order to simplify the mathematical implementation of the aging model into our previous electrochemical and thermal model, ${ }^{17}$ Safari et al.'s model was modified. The electric potential of the negative electrode, $\phi_{s, n}$, is considered to be given by the sum of the thermodynamic equilibrium potential, $U_{n}$, the electrode overpotential, $\bar{\eta}_{n}$, and the ohmic drop due to the SEI film, as classically performed in SP approaches. ${ }^{20,21}$

$$
\phi_{s, n}=U_{n}+\bar{\eta}_{n}+\frac{\delta_{S E I}}{\kappa_{S E I}} i_{t}
$$


To simplify the model structure, the total current density, $i_{t}$, can be expressed as the ratio between the applied current intensity, $I$, and the electroactive surface of the negative electrode, $S_{n}$, defined as: ${ }^{20-23}$

$$
S_{n}=3 \varepsilon_{s, n} \delta_{n} A / R_{s, n}
$$

so that:

$$
i_{t}=\frac{I}{S_{n}}=\frac{I R_{s, n}}{3 \varepsilon_{s, n} \delta_{n} A}
$$

Considering a classical Arrhenius law, where $E_{a_{-} k}$ is the activation energy of the rate constant of the side reaction, to account for the dependence on the internal temperature, $T_{\text {int }}$, on the one hand, and reintroducing the equilibrium potential of the solvent reduction, $U_{s}$, on the other hand, Eq. 15 can be written as follows:

$$
i_{s}=-i_{s}^{0} \exp \left(\frac{-E_{a_{-} k}}{R}\left(\frac{1}{T_{\mathrm{int}}}-\frac{1}{T_{\text {ref }}}\right)\right) \exp \left(\frac{\beta F}{R T_{\mathrm{int}}} \frac{\delta_{S E I}}{\kappa_{S E I}} \frac{I}{S_{n}}\right) \exp \left(-\frac{\beta F}{R T_{\mathrm{int}}}\left(\phi_{s, n}-U_{s}\right)\right)
$$

Eq. 22 presents the impact of stress factors, such as the internal temperature and the applied current intensity. The impact of the cell state-of-charge, $\mathrm{SOC}_{\text {bat }}$, is integrated in the exchange current density of the side reaction, $i_{s}^{0}$. According to the law of mass action for the solvent reduction (Eq. 14), $i_{s}^{0}$ can be expressed as a function of the concentration of lithium, $c_{s, n}^{s}$, at the surface of the single particle representing the negative electrode: ${ }^{24,25}$

$$
i_{s}^{0}=2 F k_{f}^{\prime} c_{s o l v}^{*}\left(c_{s, n}^{s}\right)^{2}=2 F k_{f}^{\prime} c_{s, n, \max }^{2} c_{s o l v}^{*}\left(\theta_{n}^{s}\right)^{2}
$$

The kinetic constant $k_{f}$ of the side reaction can be expressed as a function of $k_{f}^{\prime}$ and $c_{s, n \text {,max }}$ :

$$
k_{f}=2 k_{f}^{\prime} \exp \left(\frac{-E_{a_{-} k}}{R}\left(\frac{1}{T_{\mathrm{int}}}-\frac{1}{T_{\text {ref }}}\right)\right) c_{s, n, \max }^{2}\left(\theta_{n}^{s}\right)^{2} \exp \left(\frac{\beta F}{R T_{\mathrm{int}}} U_{s}\right)
$$

Eq. 23 gives interesting information about the impact of the state-of-charge of the negative electrode on the rate of the side reaction. The higher the SOC of the cell, the higher 
the normalized inserted $\mathrm{Li}$ ion concentration at the surface of the negative electrode, $\theta_{n}^{s}$, and, therefore, the higher the rate of the side reaction.

Assuming that the $\mathrm{Li}$ ions contained in the negative electrode contribute to the side reaction after delithiation, the variation with time of the available charge, $Q_{s}$, of the cyclable Li can be expressed as:

$$
\frac{d}{d t} Q_{s}=S_{n} i_{s}
$$

The initial condition of Eq. 25 is given by the maximal cyclable Li charge in the negative electrode that can be expressed as a function of the maximal concentration of $\mathrm{Li}$ ions, $c_{s, n, \max }$, and the stoechiometric coefficients at $0 \%$ and $100 \%$ SOC of the negative electrode, $x_{0 \%}$ and $x_{100 \%}$ :

$$
Q_{s, \text { init }}=\varepsilon_{s, n} F \delta_{n} A c_{s, n, \max }\left|x_{100 \%}-x_{0 \%}\right|
$$

Eqs. 25 and 26 have been used to quantify the capacity fade due to the cyclable Li ions consumption and update the inserted $\mathrm{Li}$ concentration, $\theta_{n}^{s}$, at the surface of the negative electrode during the aging simulations, from the ratio $Q_{S} / Q_{s, \text { init }} \cdot{ }^{25}$

Regarding the predictability of power loss, the rise of the cell impedance is generally correlated to the SEI growth rate. ${ }^{24,25}$ According to Eqs. 18 and 20, the electrical resistance, $R_{S E I}$, of the layer increases with the growth rate as follows:

$$
\frac{d}{d t} R_{S E I}=\frac{1}{\kappa_{S E I} S_{n}} \frac{d}{d t} \delta_{S E I}=-\frac{1}{\kappa_{S E I} S_{n}} \frac{i_{s} M_{S E I}}{2 F \rho_{S E I}}=-\frac{i_{S} M_{S E I}}{6 F \rho_{S E I}} \frac{R_{s, n}}{\varepsilon_{s, n} \delta_{n} A \kappa_{S E I}}
$$

Since the SEI layer was not considered in the nominal electrochemical and thermal model presented in Ref. 17, the additional overpotential term, $-I \delta_{S E I}(t) / \kappa_{S E} S_{n}$, has to be integrated into Eq. 8 to account for the impact of the growing layer on the power performances of the cell as the system ages. 
As the SEI layer grows inside the porosity of the negative electrode, the available volume fraction of the electrolyte, $\varepsilon_{e, n}$, decreases. Under the strong assumption that the layer grows uniformly on a spherical particle, a simple volume balance gives the evolution of the electrolyte volume fraction in the negative electrode as a function of the SEI thickness:

$$
\varepsilon_{e, n}(t)=1-\varepsilon_{f, n}-\varepsilon_{s, n}\left(1+\frac{3 \delta_{S E I}(t)}{R_{s, n}}\right)
$$

where $\varepsilon_{f, n}$ is the volume fraction of the filler in this electrode. This equation assumes that the electrolyte volume fraction in the SEI film can be neglected $\left(\varepsilon_{S E I}=5 \%\right.$ in Ref. 2). Derivation of Eq. 28 gives the dynamic evolution of the lumped electrode porosity:

$$
\frac{d}{d t} \varepsilon_{e, n}=-\frac{3 \varepsilon_{s, n}}{R_{s, n}} \frac{d}{d t} \delta_{S E I}=+\frac{i_{s} M_{S E I}}{2 F \rho_{S E I}} \frac{3 \varepsilon_{s, n}}{R_{s, n}}
$$

Eq. 28 can be used to introduce the concept of critical SEI thickness, $\delta_{S E I}^{c}$, which corresponds to a complete filling of the electrode porosity $\left(\varepsilon_{e, n}=0\right)$. $\delta_{S E I}^{c}$ can be simply expressed as a function of the design parameters, $\varepsilon_{f, n}$ and $\varepsilon_{s, n}$, and radius, $R_{s, n}$, of the SP representing the negative electrode:

$$
\delta_{S E I}^{c}=\left(\frac{1-\varepsilon_{f, n}}{\varepsilon_{s, n}}-1\right) \frac{R_{s, n}}{3}
$$

Thus, the design of the negative electrode, which depends on the battery typology (power or energy application), can significantly change and impact the value of the critical SEI thickness. From the values of the design parameters in Eq. 30 reported in Refs. 26 and 27, the values of $\delta_{S E I}^{c}$ for a HEV and a PHEV Li-ion cells are respectively $3.25 \mu \mathrm{m}$ and $3.75 \mu \mathrm{m}$, which can be compared to the values of the electrode thickness, $44.6 \mu \mathrm{m}$ and $97.1 \mu \mathrm{m}$ respectively. The effective transport properties of the electrolyte in the porous negative electrode will be impacted as well, since the effective electrolyte conductivity, $\kappa_{n}^{\text {eff }}$, could tend to zero, according to: 


$$
\kappa_{n}^{e f f}=\kappa\left(c_{e}\right) \varepsilon_{e, n}^{\text {Brugg,n }}=\kappa\left(c_{e}\right)\left\{1-\varepsilon_{f, n}-\varepsilon_{s, n}\left(1+\frac{3 \delta_{S E I}(t)}{R_{s, n}}\right)\right\}^{\text {Brugg, }, n}
$$

where Brugg, $n$ is the Bruggman exponent of the negative electrode.

The electrolyte resistance of the whole cell, $R_{\text {ohm }}$, the expression of which is given in the last term of Eq. 7 (Eq. 39 in Ref. 17), will then increase with aging:

$$
R_{\text {ohm }}(t)=\frac{1}{2 A}\left(\frac{\delta_{n}}{\kappa\left(c_{e}\right)\left\{1-\varepsilon_{f, n}-\varepsilon_{s, n}\left(1+\frac{3 \delta_{S E I}(t)}{R_{s, n}}\right)\right\}^{\text {Brugg, }, n}}+2 \frac{\delta_{\text {sep }}}{\kappa_{\text {sep }}^{\text {eff }}}+\frac{\delta_{p}}{\kappa_{p}^{\text {eff }}}\right)
$$

Moreover, the effective diffusivity, $D_{e, n}^{e f f}$, of $\mathrm{Li}$ ions in the negative electrode porosity will decrease with aging, as expressed by:

$$
D_{e, n}^{e f f}=D_{e} \varepsilon_{e, n}^{\text {Brugg,n }}=D_{e}\left\{1-\varepsilon_{f, n}-\varepsilon_{s, n}\left(1+\frac{3 \delta_{S E I}(t)}{R_{s, n}}\right)\right\}^{\text {Brugg, } n}
$$

The above aging model integrates both SOC and temperature effects. The SOC effect is taken into account through the expression of the exchange current density of the side reaction (Eq. 22-23) while the thermal impact is integrated through Arrhenius law in both the current density of the side reaction (Eq. 22) and the solvent diffusion coefficient defined as:

$$
D_{\text {solv }}=D_{\text {solv }}^{0} \exp \left(\frac{-E_{a_{-} D_{\text {solv }}}}{R}\left(\frac{1}{T_{\text {int }}}-\frac{1}{T_{\text {ref }}}\right)\right)
$$

In this preliminary modeling work, the degradation mechanisms occurring at the positive electrode are not considered. To briefly summarize the main mechanisms and hypotheses of the present battery aging model designed for the $\mathrm{LiFePO}_{4}$-graphite technology, a single source of aging is considered with the solvent reduction reaction occurring at the negative electrode interface. The consumption of cyclable Li ions from the negative electrode proceeds via mass transport of solvent molecules through the SEI layer by diffusion and convection (Eq. 17) leading to capacity loss (Eq. 25). No modification of the electrolyte 
concentration is considered in this work; only the volume and effective transport properties of the electrolyte in the porosity of the negative electrode change during aging. Indeed, because of the formation of insoluble products, the SEI thickness increases (Eq. 18), gradually filling the porosity of the negative electrode and increasing the film resistance (Eq. 27). The porosity decrease impacts the effective transport properties of the electrolyte leading to a decrease of the effective diffusion coefficient (Eq. 33) and a rise of ohmic resistance (Eq. 32), resulting in a power loss of the system. At the limiting case where the SEI layer reaches its critical thickness (Eq. 30), it is assumed that no access to the active material is possible anymore. This is the End-Of-Life state of the battery system.

\section{Experimental calibration and validation of the aging model}

Calibration of capacity fade on calendar tests. - The SIMCAL project, which is funded by the French Research National Agency, provides interesting databases on the capacity and power evolutions during calendar aging of different Li-ion cell technologies. ${ }^{28,29}$ In the database concerning the $\mathrm{LiFePO}_{4}$-graphite system under study (high-power cell of nominal capacity 2.3 Ah, A123 Systems ANR26650m1), stress factors like temperature and SOC are being investigated for nine calendar operating conditions: three temperatures $\left(30^{\circ} \mathrm{C}, 45^{\circ} \mathrm{C}\right.$, and $\left.60^{\circ} \mathrm{C}\right)$ and three SOC (30\%, 65\%, and $\left.100 \%\right) .{ }^{28}$ Experimental repeatability is obtained by testing three cells for each operating condition. The mean initial capacity, determined for the 27 cells with a 1C discharge protocol, was 2.175 Ah. The results of capacity loss clearly show aging activation for increasing values of temperature and SOC (Fig. 2), which is in accordance with results reported in the literature. ${ }^{12}$

With the simulation parameters presented in Table IV and the thermal parameters of the cell given in Ref. 17 (Table V), a satisfactory agreement is obtained on calendar tests, as shown in Fig. 2, after adjusting the solvent diffusion coefficient and kinetic rate of the side 
reaction. It should be noted that the values of $D_{\text {solv }}^{0}$ in Table IV and of the kinetic rate derived from Eq. $24\left(k_{f}=6.85 \times 10^{-11} \mathrm{~m} . \mathrm{s}^{-1}\right.$ at $\left.298 \mathrm{~K}\right)$, are in good agreement with those reported in Delacourt and Safari's work. ${ }^{30-31}$ The activation energy values of the solvent diffusivity and the side reaction rate constant have also been manually adjusted to fit the experimental data at $30^{\circ} \mathrm{C}, 45^{\circ} \mathrm{C}$, and $60^{\circ} \mathrm{C}$. The values obtained for these parameters are in good agreement with reported values. $^{32,33}$ Interestingly, a dispersion can be observed for the tests performed at $60^{\circ} \mathrm{C}$. At this temperature, other aging mechanisms could occur.

Calibration of capacity fade on cycling tests. - The present electrochemical and thermal aging model is used in this section to simulate the results of the extensive experimental work performed by Wang et al. in which the mean capacity loss and the mean skin temperature measured during accelerated cycling tests at $\mathrm{C} / 2,2 \mathrm{C}, 6 \mathrm{C}$, and $10 \mathrm{C}$ regimes and $15^{\circ} \mathrm{C}, 25^{\circ} \mathrm{C}$, $45^{\circ} \mathrm{C}$, and $60^{\circ} \mathrm{C}$ temperatures are reported. ${ }^{7}$ To run the cycling simulations without modifying the radius of the active particles as performed in Ref. 17, the diffusion coefficient in the positive electrode was set to a value of $5.9 \times 10^{-18} \mathrm{~m}^{2} / \mathrm{s}$ as indicated in Table IV. Based on their experimental results, Wang et al. developed a semi-empirical aging model for capacity fade prediction to mathematically describe the whole set of tests. Its originality is based on the introduction of current intensity (C-rate in Eq. 7 of Ref. 7) into the capacity fade equation. Considering the rather important dispersion in their values of capacity loss for the two cells tested, the curves derived from their semi-empirical model were used in this paper, instead of their raw values of capacity loss, as a reference for calibrating our physics-based model. The complete database was carefully analyzed and attention was paid to the thermal conditions during the endurance cycling tests in which the convective conditions were not clearly defined. To simulate appropriate electrical and thermal conditions during the aging tests, a preliminary set of parametric simulations was performed to determine the approximated 
Newton convective coefficient, $h_{\text {conv }}$, required to fit the reported mean skin temperatures. For the skin temperature values missing in Ref 7 (Table 2), it was considered that the skin temperature increase due to current cycling should remain within a range of $2-3^{\circ} \mathrm{C}$ above the test temperature. Once the sixteen $h_{\text {conv }}$ values determined through these preliminary simulations, the numerical aging tests were carried out. With the set of parameters used for the calendar simulations, the first simulations in cycling mode presented large deviations in capacity loss compared to the experimental values. The higher the current intensity, the higher the deviations. In order to keep a similar set of parameters for both calendar and cycling conditions, only the consumption and mass transport of the solvent were modified in the simulations presented in Fig. 3. The value of the solvent reduction kinetic rate $k_{f}^{\prime}$ for the cycling mode was set at 120 times the value of $k_{f}^{\prime}$ determined for the calendar mode. This factor of 120 has no physical meaning; it was manually tuned to help fitting the Wang et al.'s cycling experimental data. ${ }^{7}$ The effective mass transport can be increased by either increasing the porosity $\varepsilon_{S E I}$ of the SEI layer, or by increasing the effective diffusion coefficient in the SEI layer. In Safari et al.'s work ${ }^{2}$, the porosity of the SEI layer is mathematically only introduced in the equations as the boundary condition for the solvent diffusion through the SEI layer. Increasing $\varepsilon_{S E I}$ with the current is a way to increase the solvent concentration at the SEI/electrolyte boundary and thereby the concentration gradient in the SEI layer. Indeed, as it is well known that the SEI film exhibits limited elasticity and is prone to crack during cycling operations, the value of the SEI porosity, $\varepsilon_{S E I}$, could have been tuned to help fitting the experimental data. However, since Eq. 17 and 18 represent the transport mechanism in a nonporous medium, and to simplify the mathematical implementation of the model developed for simulation purposes, it was decided to fit Wang et al's experimental data ${ }^{7}$ by adjusting the effective diffusion coefficient with the current intensity. The values of the solvent diffusion coefficient, $D_{\text {solv }}^{0}$, as a function of the current intensity, which give a good agreement between 
our simulations of capacity loss and those reported by Wang et al. ${ }^{7}$, and maybe traduce the impact of mechanical stress at high C-rates, are shown in Fig. 4. Under these conditions, a good agreement is obtained between the values of capacity loss simulated with our simple electrochemical and thermal aging model and those simulated with Wang et al.'s semiempirical model.

It is well known that the current modifies the quality and the passivating role of the SEI during cycling, so that the modification of the consumption and mass transport of the solvent in this purely electrochemical and thermal model could be a way to represent another well reported aging mechanism, namely the SEI mechanical fracture due to particle cracks during battery cycling. For example, Wachtler et al. mentioned the cracking of the SEI of different negative electrodes used in Li-ion systems as a possible aging mechanism leading to capacity and power fades of carbonaceous, Li metal and alloys negative electrodes under cycling operating conditions. ${ }^{34}$ Very recently, Deshpande et al. developed a mathematical model of capacity fade for battery cycle life simulation, integrating crack propagation of the spherical carbonaceous particles and SEI growth on the cracked surface. ${ }^{35}$

Mechanical stress within the active particles as insertion/deinsertion occurs, is then a first possible hypothesis to explain the results. Indeed, Li-ion insertion into graphite particles during charge increases the lattice volume, which may partially break the particles and alter the SEI film passivity by exposing newly formed electroactive carbon surface on which the electrolyte can react. This hypothesis has been widely mentioned in the literature and theoretically modeled. Garcia et al. $^{36}$ developed a model coupling electrochemical and mechanical phenomena to quantify electrochemically induced stresses in Li-ion battery electrodes. They showed that large stresses develop at high-rate discharge and contribute to mechanical failure of the electrodes. Later, combining diffusion-controlled kinetics and fracture mechanics, Zhao et al. ${ }^{37}$ investigated how material properties, particle size, and 
discharge rate affect the electrode fracture in lithium-ion batteries. Renganathan et al. also analyzed the impact of current intensity and design parameters (electrodes thickness, particle radius, and porosity) on the fracture phenomenon of carbon particles. ${ }^{38}$ According to their model, the stress values within the carbon particles were higher than the tensile yield strength (30 MPa), leading to the presence of cracks above a 6C rate.

Another possible mechanism of SEI degradation was proposed by Ning et al. ${ }^{39}$ who studied the aging of $\mathrm{LiNiAlCoO}_{2}$-graphite lithium batteries in high discharge rate operating conditions. According to them, the build-up of pressure due to the gaseous products released by the side reaction during cycling might stretch and damage the SEI film. As a consequence, Li ions and electrolyte could go through the newly formed cracks and react with the carbon particles. The SEM pictures in Ref. 39 clearly demonstrate the presence of cracks at 2C and 3C regimes on negative carbonaceous electrodes.

For both above-mentioned mechanical phenomena (Li-insertion induced stress or pressure build-up), the capacity fade is accelerated because of the progressive increase in the active surface where the electrolyte can be reduced and consume cyclable lithium. According to the experimental observations in Ref. 39, the theoretical mechanical models in Refs. 36-38, and the numerical results obtained in this section, our aging model seems too simple to properly take into account the complexity of the physical, chemical, and mechanical phenomena occurring in the battery, especially at high C-rates. Without tuning the reaction and mass transport parameters, $k_{f}^{\prime}$ and $D_{\text {solv }}^{0}$, of the solvent reduction, the model simulations are in good agreement with experimental data only for C-rates lower than C/2. At higher C-rates, a fracture model should be integrated to take into account the mechanical phenomena.

Recently, Delacourt and Safari also simulated the capacity fade of the $\mathrm{LiFePO}_{4}$ graphite battery under study in this paper for conventional cycling and driving complex duty cycles of EV. ${ }^{30-31}$ They obtained good results with their aging model, which takes into 
account the loss of active material of the negative electrode for cycling operating conditions, in addition to the loss of cyclable $\mathrm{Li}^{+}$ions. In their work, this loss of active material was attributed to the isolation of active material particles due to mechanical phenomena occurring during cycling operations and the impact of current intensity on the decrease in the volume fraction of active material in the negative electrode, $\varepsilon_{s, n}$, was described empirically.

Our simplified electrochemical and thermal aging model does not take into account the effect of mechanical stress, nor the loss of active material occurring during cycling operating conditions. However, it shows satisfactory results on both calendar and cycling operating conditions at different temperature, SOC, and current values, and can be easily used to simulate complex cycling profiles such as driving duty cycles for which the current applied cannot be predicted. It is now used in the rest of the paper to investigate correlations between capacity fade and power loss.

Investigation of correlations between capacity fade and power loss on cycling tests.Literature on aging studies of $\mathrm{LiFePO}_{4}$-graphite batteries offers little information about the correlations between power fade and capacity loss. In order to investigate energy and power losses, cycling tests coupled with EIS experiments were performed at IFPEN on three A123 Systems cells of 2.3 Ah capacity, in particular with the C/2 regime protocol defined by Wang et al. $^{7}$ to compare their database at this regime with our results. During the cycling tests, accelerated aging was investigated at high temperature $\left(60^{\circ} \mathrm{C}\right)$ and, between two aging periods, check-up protocols were performed at $20^{\circ} \mathrm{C}$ to determine the residual capacity at a 1C discharge regime and measure the impedance spectra at equilibrium $(I=0)$ and different SOC. Then, a fitting procedure was used to determine the impedance parameters of the equivalent electrical circuit as a function of aging. 
As illustrated in the Nyquist diagram of a typical impedance spectrum (Fig. 5), several resistive contributions corresponding to different electrochemical phenomena can be distinguished in separate frequency ranges. As described in our previous work, the resistance, $R_{o h m}$, given by the intercept of the impedance curve with the real axis in the Nyquist diagram, is considered to represent the static electrolyte resistance given in Eq. 32. The high-frequency capacitive loop contains the kinetic contributions of the SEI film and of the negative and positive electrodes. Depending on parameters such as the SEI thickness and cell temperature, two separate loops may sometimes appear, ${ }^{40-42}$ but in many cases, as in Fig. 5, a single capacitive loop can be observed, either because the resistance of the film is small, or because the time constants involved in the two loops are very close. In that case, the high-frequency semi-circle diameter, $R_{S C}$, is assumed to represent the sum of the charge-transfer resistances at both electrodes, $R_{c t}^{n}$ and $R_{c t}^{p}$, and of the SEI layer resistance, $R_{S E I}$. As EIS measurements were performed at a zero mean current, Eq. 3 can be linearized to express the charge-transfer resistance of the positive and negative electrodes:

$$
R_{c t}^{p}=\frac{R T}{F i_{0, p} S_{p}} \quad \text { and } \quad R_{c t}^{n}=\frac{R T}{F i_{0, n} S_{n}}
$$

As indicated above, the degradation mechanisms occurring at the positive electrode are not considered in this work so that the film resistance on the positive electrode is neglected and $R_{S C}$ is considered to be given by:

$$
R_{S C}=R_{c t}^{p}+R_{c t}^{n}+R_{S E I}=\frac{R T}{F i_{0, p} S_{p}}+\frac{R T}{F i_{0, n} S_{n}}+\frac{\delta_{S E I}}{\kappa_{S E I} S_{n}}
$$

where $S_{p}=3 \varepsilon_{s, p} \delta_{p} A / R_{s, p}$ and $S_{n}$ (Eq. 20) are the electro-active surface areas of the positive and negative electrodes, respectively. It should be noted that unlike in Ref. 17, where the exchange current density for the charge-transfer reaction on the negative electrode, $i_{0, n}$, was taken to be $0.5 \mathrm{~A} / \mathrm{m}^{2}$ a detailed experimental analysis of impedance data revealed that a proper 
value for this parameter is around $7.5 \mathrm{~A} / \mathrm{m}^{2}$, so that Eq. 36 gives an initial value of $R_{S C}$ before aging of $3.2 \mathrm{~m} \Omega$ with $i_{0, p}=0.05 \mathrm{~A} / \mathrm{m}^{2}$.

Finally, $R_{W}$ is a representation of the different diffusion phenomena occurring in the cell. Based on the shape of the impedance spectrum, the equivalent circuit composed of a resistance, $R_{\text {ohm }}$, in series with an inductance and two resistances in parallel with two constant phase elements, as represented in Fig. 5, was selected to calculate the $R_{o h m}$ and $R_{S C}$ values from the experimental impedance spectra.

From a practical engineering point of view, correlations between capacity loss and resistance increase can be of valuable help to diagnose the state of health of battery packs, especially in applications where no capacity tests can be carried out to determine the residual capacity of the battery, as for HEV applications for instance. With the assumptions of the present model (loss of cyclable Li), integrating Eqs. 25 and 27, and using Eq. 20, allow the capacity loss of the battery, $C_{L O S S}(t)$ to be expressed as a function of the $R_{S C}$ increase, $\Delta R_{S C}$, which is considered to be equal to the $R_{S E I}$ increase (Eq. 36):

$$
C_{\text {LOSS }}(t)=100 \times \frac{Q_{s, \text { init }}-Q_{s}(t)}{Q_{s, \text { init }}}=100 \times \frac{2 F \rho_{S E I} \kappa_{S E I}}{M_{S E I}}\left(\frac{S_{n}{ }^{2}}{Q_{s, \text { init }}}\right) \Delta R_{S C}
$$

Equation 37 can be used as an interesting indicator to determine the capacity loss from the measurement of the increase in the diameter of the semi-circle resistance, $\Delta R_{S C}$, in the Nyquist representation. This relation points out the influence on the capacity loss of both the design parameters of the negative electrode and the physical properties of the SEI layer.

As previously mentioned, the present aging model provides direct analytic correlations of the thickness of the SEI layer with the ohmic resistance (Eq. 32), and with the highfrequency semi-circle resistance (Eq. 36). These two correlations are now analyzed for the cycling experimental data. As can be observed in Fig. 6a, the mean value of the capacity loss measured on the three cells is in good agreement with the data published by Wang et al. and 
with the capacity loss simulated by our aging model. With the same initial value of $5 \mathrm{~nm}$, the model calculates a SEI thickness of $400 \mathrm{~nm}$ after 1500 cycles (Fig. 6b). In order to fit the simulated and experimental values of the ohmic resistance as a function of the cycle number (Fig. 6c) the Bruggman coefficient was set to 1.3 to start the simulation from the initial value $R_{\text {ohm }}=7.95 \mathrm{~m} \Omega$. It should be noticed that values of $R_{o h m}$ ranging between 7.5 and $8.7 \mathrm{~m} \Omega$ may be found in the literature for the A123 Systems cells of capacity $2.3 \mathrm{Ah}$, which may be explained by differences in connectors of the experimental set-up, differences in the manufacturing processes, or by different levels of aging when the tests were carried out. As a consequence, the change in the value of Brugg, $n$ in Eq. 32 has no physical meaning; it is used to adjust the initial value of $R_{o h m}$. Considering the scatter in the measured $R_{S C}$ values, the evolution of the simulated $R_{S C}$ values with aging is satisfactory (Fig. 6d). It should be noticed that different orders of magnitude may be found in the literature for the value of $\kappa_{\text {SEI }}$, ranging from $3.79 \times 10^{-7} \mathrm{~S} / \mathrm{m}$ in Ref. 20 to $1 \mathrm{~S} / \mathrm{m}$ in Ref. 24 for various Li-ion batteries.

In summary, two parameters were adjusted in this section to get a good agreement between capacity and power loss simulations and experimental data, namely the Bruggman exponent and the SEI ionic conductivity. Slight modifications of the Bruggman exponent were necessary to fit the initial value of ohmic resistance, $R_{\text {ohm }}(0)$. Indeed, some dispersion in the experimental initial values of $R_{o h m}$ in the calendar and cycling tests may be observed. They can be due to differences in connectors or by intrinsic cell manufacturing dispersion. Another possible explanation could be different aging levels at the initial characterization test. The simulated evolution of $R_{\text {ohm }}$ is not in excellent agreement with the experimental values. This could be explained by a possible consumption of Li-ions from the electrolyte phase, as will be discussed in the following section. On the other hand, the SEI ionic conductivity, $\kappa_{S E I}$, was adjusted to follow the evolution of the semi-circle resistance, $R_{S C}$, with aging. 
Validation of the modified electrochemical and thermal aging model.— The aging model was calibrated above for both calendar and simple galvanostatic cycling endurance tests. Capacity and power loss correlations were proposed and EIS experimental data validated the assumptions and interpretations of impedance spectra. The present aging model could then be used for BMS of portable devices since simple current profiles are generally used for such applications. However, the present aging model was designed to be used in BMS applications related to the transportation field, in which current profiles can be complex with dynamic pulse solicitations in charge and discharge. Thus, in order to validate the modified aging model on complex vehicle duty profiles, specific experiments were carried out at IFPEN with the A123 Systems cell of 2.3 Ah capacity. Vehicle simulations platforms were used to define the mission profile of a PHEV with different driving modes. A zero-emission vehicle (ZEV) mode with a pure electric driving operation was considered. The macro-cycle profile, composed of four driving profiles separated by a 1-hour rest period and a recharge, was experimentally programmed. Four macro-cycles were repeated during a day period at a temperature of $45^{\circ} \mathrm{C}$. Every thirty-two macro-cycles a capacity check and EIS measurements were carried out at $23^{\circ} \mathrm{C}$. The initial SOC of the macro cycle was $90 \%$ and the DOD was approximately 70\%. As can be seen in Fig. 7, the ZEV macro-cycle contains both calendar periods, a classic galvanostatic recharge and four dynamic vehicle-duty-cycle solicitations. The intensity of the current pulses during the driving mode was within the calibration range of the model, namely 9C.

In this validation test, the Bruggman exponent of the negative electrode, Brugg,n, was set to 1.5 to obtain the experimental initial value of $8.3 \mathrm{~m} \Omega$ for $R_{o h m}(0)$, and $\kappa_{\text {SEI }}$ was set to $17 \times 10^{-5} \mathrm{~S} / \mathrm{m}$, as for the cycling tests (Fig.6). As can be observed in Fig. 8, the model fits well the experimental data for both residual capacity and resistances. The capacity loss, of about $8 \%$, was lower than in the calendar test performed at $45^{\circ} \mathrm{C}$ and a SOC of $100 \%$ (Fig. 2), 
indicating that cycling tests are more severe for accelerated aging than calendar tests. This is in line with the aforementioned analysis concerning the impact of current intensity on capacity fade evolution due to particle cracks during battery cycling (Fig. 3). It should also be noted that the value of capacity loss after 3 months of complex cycling at $45^{\circ} \mathrm{C}$ is in good agreement with the value of $8 \%$ measured in similar temperature and cycling conditions and reported in Fig. 13 in Ref. 30. In contrast with $R_{\text {ohm }}$ that increased by $12 \%$, roughly as in Fig. $6, R_{S C}$ increased only by a factor of $9 \%$, in accordance to the lower capacity fade observed in Fig. 8.

Interestingly, similar trends and correlations can be observed in the evolutions of residual capacity and $R_{S C}$ in Figs.6 and 8. This will be discussed in the next section, in which a theoretical state-of-health indicator based on $R_{S C}$ measurement will be defined. Despite strong hypotheses (loss of cyclable Li without loss of active material), our simple modified electrochemical and thermal aging model gives good results for calendar aging, and in conventional and complex cycling operating conditions. As the model does not integrate equations governing mechanical degradation, it is reminded that the empirical function accounting for the impact of current intensity on aging (Fig. 4) was used to keep the model structure as simple as possible even if this function has no physical meaning. Addition of theoretical laws of solid mechanics, as performed in Ref. 35, will be added in a future work.

\section{Discussion on the power loss and capacity fade correlations}

The simplified aging model proposed in the present work is able to simulate both capacity fade and power loss of the $\mathrm{LiFePO}_{4}$-graphite battery under study. Two parameters, Brugg, $n$ and $\kappa_{S E I}$, were determined by fitting the resistances $R_{o h m}$ and $R_{S C}$ to the experimental values given by the EIS technique. Despite the possible mechanism of particle fracture under high rate cycling, the particle radius, $R_{s, n}$, and the electroactive surface, $S_{n}$, of the negative electrode were not modified to fit the experimental data. The values of $R_{\text {ohm }}$ and of the SEI 
resistance, $R_{S E I}$, given respectively by Eq. 32 and the expression $\delta_{S E I} /\left(\kappa_{S E I} S_{n}\right)$, are strongly dependent on the physical properties of the porous medium and of the SEI film. Figure 9 shows the evolution of these two resistances as a function of the SEI thickness. As the SEI thickness increases, the ohmic resistance can drastically increase, depending on Brugg, $n$. The Bruggman exponent plays an important role on both the initial value and the dynamic evolution of $R_{o h m}$ as the SEI film thickens. As described in Refs. 43 and 44, the Bruggman exponent can be correlated to the tortuosity of the electrode. Decreasing the tortuosity, and thus the Bruggman exponent, improves the performances of the electrode. On the other hand, as illustrated in Fig. $9 \mathrm{~b}$, the value of the SEI ionic conductivity, $\kappa_{S E I}$, can significantly impact the SEI resistance and, therefore, the power performance of the battery. High ionic conductivity of the SEI is essential to achieve good power performance of carbon-based Liion technologies.

Concerning the resistance $R_{\text {ohm }}$, two possible scenarios about the origin of the $\mathrm{Li}^{+}$ions consumed by the side reaction can be discussed from Eq. 32. Indeed, these ions may come from the active $\mathrm{Li}^{+}$ions contained in the negative electrode or from the electrolyte. In the former case, as proposed by Ramasamy et al. for calendar operating conditions, ${ }^{24}$ the delithiation reaction at the negative electrode is considered to be the reaction providing electrons consumed by the side reaction (Eq. 14), so that the concentration of $\mathrm{Li}^{+}$in the electrolyte phase, $c_{e}$, is not modified and the evolution of $R_{o h m}$ is controlled by the porosity modification. In the latter case, the active $\mathrm{Li}^{+}$ions in the electrolyte are partially consumed. This may occur especially in cycling operating conditions when fractures of particles create newly formed electroactive surface at which the electrolyte reacts. Indeed, as the battery is charged and discharged, concentration gradients in the electrolyte phase are developed in the electrode porosities. During the discharge of the battery, the delithiation reaction at the negative electrode provides $\mathrm{Li}^{+}$ions to the electrolyte phase that will diffuse to the positive 
electrode where they are consumed by the lithiation reaction. In this case, the $\mathrm{Li}^{+}$ions from the negative electrode can be used for the side reaction, as assumed by Ramasamy et al. ${ }^{24}$ In contrast, during the charge of the battery, the lithiation reaction at the negative electrode consumes $\mathrm{Li}^{+}$ions from the electrolyte at the same reactive interface as the side reaction. In this case, it is likely that $\mathrm{Li}^{+}$ions from the electrolyte are consumed by the side reaction, which modifies the $\mathrm{Li}^{+}$concentration in the electrolyte phase and leads to a decrease in the electrolyte conductivity, $\kappa$. In this particular case, the evolution of $R_{\text {ohm }}$ is controlled by both the porosity modification and the decrease in electrolyte concentration.

\section{Conclusions and perspectives}

With the hypotheses of SEI layer growth by solvent diffusion and porosity modification of the negative carbonaceous electrode, a model of Li-ion battery aging by loss of cyclable $\mathrm{Li}^{+}$ions was developed on the basis of the nominal electrochemical and thermal model presented in Ref. 17. This model was validated for a commercial $\mathrm{LiFePO}_{4}$-graphite Liion cell on both calendar and cycling endurance experimental data. Its theoretical limits about capacity fade prediction were discussed by analyzing the differences between simulations and experimental data for cycling operating conditions. Empirical correlations were introduced in the model to help fitting the experimental that are influenced by mechanical degradation phenomena at high C-rates, by considering that the solvent diffusion coefficient $D_{\text {solv }}^{0}$ depends on current intensity and that the kinetic rate of solvent reduction $k_{f}^{\prime}$ depends on the type of aging, calendar or cycling. This empirical dependence was implemented in order to keep the model simple without introducing equations modelling the mechanical degradation phenomena. Thus, the capacity loss simulated in this preliminary work theoretically takes into account loss of cyclable lithium for calendar aging and empirically lumps both loss of cyclable lithium and other degradation mechanisms for cycling operating conditions. 
Mathematical refinements of the present model to theoretically account for additional aging mechanism, such as the loss of active material at the negative electrode that was not considered in this work, will be the subject of future investigations and model developments.

Another important result in this paper concerns the development of theoretical correlations between power loss and capacity fade that were experimentally validated with endurance tests and EIS data and allowed a theoretical state-of-health indicator based on $R_{S C}$ measurement to be defined. This model-based aging indicator needs further validation on experimental data to determine whether the correlation in Eq. 37 is really applicable in practical conditions. Moreover, thanks to the introduction of the porosity filling mechanism, a new set of equations was presented and used to correlate the evolution of the ohmic resistance to the SEI thickness. This will be used in a future work to shed light into possible mechanisms leading to the end-of-life of $\mathrm{LiFePO}_{4}$-graphite $\mathrm{Li}$-ion batteries.

\section{Acknowledgements}

The authors would like to thank the SIMCAL project, which is funded by the French Research National Agency, for providing interesting databases on the capacity evolutions during calendar aging of the Li-ion cell under study. 


\section{References}

1. R. Spotnitz, J. Power Sources, 113, 72 (2003).

2. M. Safari, M. Morcrette, A. Teyssot, and C. Delacourt, J. Electrochem. Soc., 156, A145 (2009).

3. A. N. Jansen, D. W. Dees, D. P. Abraham, K. Amine, and G. L. Henriksen, J. Power Sources, 174, 373 (2007).

4. L. Gireaud, S. Grugeon, S. Laruelle, B. Yrieix, and J. M. Tarascon, Electrochem. Commun., 8, 1639 (2006).

5. Q. Zhang and R. E. White, J. Power Sources, 173, 990 (2007).

6. M. Broussely, in Advances in Lithium-Ion Batteries, W. A. van Schalkwijk and B. Scrosati, Editors, p. 393, Kluwer Academic/Plenum Publishers, New York (2002).

7. J. Wang, P. Liu, J. Hicks-Garner, E. Sherman, S. Soukiazian, M. Verbrugge, H. Tataria, J. Musser, and P. Finamore, J. Power Sources, 196, 3942 (2011).

8. J.B. Gerschler, H. Witzenhausen, F. Hust, and D. U.Sauer, Three-Dimensional Performance and Lifetime Model for Lithium-Ion Batteries, $25^{\text {th }}$ Electric Vehicle Symposium, Shenzhen, China (2010).

9. J. Newman and K. E. Thomas-Alyea, Electrochemical Systems, third edition, John Wiley \& Sons Inc., Hoboken, New Jersey (2004).

10. S. Santhanagopalan, Q. Guo, P. Ramadass, and R. E. White, J. Power Sources, 156, 620 (2006).

11. J. Christensen and J. Newman, J. Electrochem. Soc., 151, A1977 (2004).

12. A. M. Colclasure, K. A. Smith, and R. J. Kee, Electrochim. Acta, 58, 33 (2011).

13. D. Danilov and P. H. L. Notten, Ageing of Li-ion Batteries: Mathematical Description, Abstract 143, 207 ${ }^{\text {th }}$ ECS Meeting, The Electrochemical Society, Vol. MA2005-1, Quebec City, Canada (2005). 
14. H. J. Ploehn, P. Ramadass, and R. E. White, J. Electrochem. Soc., 151, A456 (2004).

15. G. Sikha, B. N. Popov, and R. E. White, J. Electrochem. Soc., 151, A1104 (2004).

16. H. Jannesari, M. D. Emami, and C. Ziegler, J. Power Sources, 196, 9654 (2011).

17. E. Prada, D. Di Domenico, Y. Creff, J. Bernard, V. Sauvant-Moynot, and F. Huet, J. Electrochem. Soc., 159, A1508 (2012).

18. J. Wang, S. Soukiazian, M. Verbrugge, H. Tataria, D. Coates, D. Hall, and P. Liu, J. Power Sources, 196, 5966 (2011).

19. M. Dubarry, V. Svoboda, R. Hwu, and B. Y. Liaw, J. Power Sources, 165, 566 (2007).

20. G. Ning, R. E. White, and B. N. Popov, Electrochim. Acta, 51, 2012 (2006).

21. G. Ning and B. N. Popov, J. Electrochem. Soc., 151, A1584 (2004).

22. B. Kenney, K. Darcovich, D. D. MacNeil, and I. J. Davidson, J. Power Sources, 213, 391 (2012).

23. S. K. Rahimian, S. Rayman, and R. E. White, J. Power Sources, 196, 10297 (2011).

24. R. P. Ramasamy, J.-W. Lee, and B. N. Popov, J. Power Sources, 166, 266 (2007).

25. P. Ramadass, B. Haran, P. M. Gomadam, R. White, and B. N. Popov, J. Electrochem. Soc., 151, A196 (2004).

26. P. Albertus and J. Newman, J. Power Sources, 183, 376 (2008) .

27. P. Albertus, J. Couts, V. Srinivasan, and J. Newman, J. Power Sources, 183, 771 (2008).

28. J. Bernard, R. Revel, The French Simcal research Network for modelling of Energy Storage System Calendar Aging in EVs and HEVs - First Results on LFP/C Power Cells, $16^{\text {th }}$ International Meeting on Lithium Batteries, The Korean Electrochemical Society, Jeju, Korea (2012).

29. M. Kassem, J. Bernard, R. Revel, S. Pélissier, F. Duclaud, and C. Delacourt, J. Power Sources, 208, 296 (2012).

30. C. Delacourt and M. Safari, J. Electrochem. Soc., 159, A1283 (2012). 
31. M. Safari and C. Delacourt, J. Electrochem. Soc., 158, A1436 (2011).

32. K. Xu and A. von Cresce, J. Mater. Chem., 21, 9849 (2011).

33. K. Xu, A. von Cresce, and U. Lee, Langmuir, 26, 11538 (2010).

34. M. Wachtler, J. O. Besenhard, M. Winter. J. Power Sources, 94, 189 (2001)

35. R. Deshpande, M. Verbrugge, Y.-T. Cheng, J. Wang, P. Liu, J. Electrochem. Soc., 159, A1730 (2012).

36. R. E. García, Y.-M. Chiang, W. C. Carter, P. Limthongkul, and C. M. Bishop, J. Electrochem. Soc., 152, A255 (2005).

37. K. Zhao, M. Pharr, J. J. Vlassak, and Z. Suo, J. Appl. Phys., 108, 073517 (2010).

38. S. Renganathan, G. Sikha, S. Santhanagopalan, and R. E. White, J. Electrochem. Soc., 157, A155 (2010).

39. G. Ning, B. Haran, and B. N. Popov, J. Power Sources, 117, 160 (2003).

40. M. D. Levi and D. Aurbach, J. Power Sources, 146, 727 (2005).

41. H. Nakahara, S.-Y. Yoon, T. Piao, F. Mansfeld, and S. Nutt, J. Power Sources, 158, 591 (2006).

42. S. Yang, J. Huo, H. Song, and X. Chen, Electrochim. Acta, 53, 2238 (2008).

43. I. V. Thorat, D. E. Stephenson, N. A. Zacharias, K. Zaghib, J. N. Harb, and D. R. Wheeler, J. Power Sources, 188, 592 (2009).

44. B. Vijayaraghavan, D. R. Ely, Y.-M. Chiang, R. García-García, and R. E. García, J. Electrochem. Soc., 159, A548 (2012). 


\section{FIGURE CAPTIONS}

Table I. 1D electrochemical equations.

Table II. Heat transfer and energy balance equations.

Table III. Equations for the aging model. ${ }^{2}$

Table IV. Parameters of the electrochemical and thermal aging model.

Figure 1. Schematic representation of the growing SEI film.

Figure 2. Model simulations of capacity loss compared to the experimental data of the calendar tests of the SIMCAL database. ${ }^{28}$

Figure 3. Model simulations of capacity loss compared to the modeled data of the cycling test database in Ref. 7.

Figure 4. Evolution of the SEI porosity as a function of current intensity in the simulations of Figs. 2 and 3 (the cells were de rated to $2 \mathrm{Ah}$ for the full cell capacity in Ref. 7).

Figure 4. Evolution of the solvent diffusion coefficient as a function of current intensity.

Figure 5. Impedance spectrum of the $\mathrm{LiFePO}_{4}$-graphite cell at $20^{\circ} \mathrm{C}$ and $50 \% \mathrm{SOC}$ and equivalent circuit used to calculate $R_{o h m}$ and $R_{S C}$.

Figure 6. a) Residual capacity simulations compared to experimental data for C/2 cycling tests at $50 \%$ DOD from an initial SOC of $100 \%$ and $60^{\circ} \mathrm{C}$, b) Simulation of the SEI layer growth as a function of cycle number, c) Comparison between simulated and measured ohmic resistance as a function of cycle number, d) Comparison between simulated and measured semi-circle resistance as a function of cycle number.

Figure 7. a) ZEV macro-cycle dynamic current profile as a function of time, b) evolution of the cell SOC during the macro-cycle.

Figure 8. a) Residual capacity simulations compared to experimental data for the ZEV cycle test at $45^{\circ} \mathrm{C}$, b) Simulation of the SEI layer growth as a function of days, c) Comparison between simulated and measured ohmic resistance as a function of days, d) Comparison between simulated and measured semi-circle resistance as a function of days.

Figure 9. a) Evolution of the ohmic resistance as a function of the Bruggman exponent and the SEI film thickness, b) Evolution of the SEI resistance as a function of the film ionic conductivity and the film thickness. 
Table I. 1D electrochemical equations.

Physical and chemical mechanism

Eq. Boundary conditions

Eq. in

Ref. 17

Solid phase conservation of $\mathrm{Li}^{+}$species

$$
\frac{\partial}{\partial t} c_{s}-\frac{1}{r^{2}} \frac{\partial}{\partial r}\left(r^{2} D_{s} \frac{\partial}{\partial r} c_{s}\right)=0
$$

$$
\begin{gathered}
\left.D_{s} \frac{\partial}{\partial r} c_{s}\right|_{r=0}=0 \\
-\left.D_{s} \frac{\partial}{\partial r} c_{s}\right|_{r=R_{s}}=\frac{j_{f}}{a_{s} F}
\end{gathered}
$$

Electrolyte phase conservation of $\mathrm{Li}^{+}$species

$$
\frac{\partial}{\partial t} \varepsilon_{e} c_{e}-\frac{\partial}{\partial z}\left(D_{e}^{e f f} \frac{\partial}{\partial z} c_{e}\right)-\left(1-t_{+}\right) \frac{j_{f}}{F}=0 \quad \text { [2] }\left.\quad \frac{\partial}{\partial z} c_{e}\right|_{z=0}=\left.\frac{\partial}{\partial z} c_{e}\right|_{z=L}=0
$$

Electrochemical $\bar{j}_{f, n}=a_{s, n} i_{0, n}\left\{\exp \left(\frac{\alpha F}{R T} \bar{\eta}_{n}^{k}\right)-\exp \left(-\frac{(1-\alpha) F}{R T} \bar{\eta}_{n}^{k}\right)\right\}$ kinetics

$$
\begin{gathered}
\bar{j}_{f, p}=a_{s, p} i_{0, p}\left\{\exp \left(\frac{\alpha F}{R T} \bar{\eta}_{p}^{k}\right)-\exp \left(-\frac{(1-\alpha) F}{R T} \bar{\eta}_{p}^{k}\right)\right\} \\
\bar{\eta}_{n}=\bar{\eta}_{n}^{k}+\bar{\eta}_{n} \text { diff } \\
\bar{\eta}_{p}=\bar{\eta}_{p}^{k}+\bar{\eta}_{p} \\
\bar{\eta}_{n}^{k}=\frac{R T}{\alpha F} \ln \left(\xi_{n}+\sqrt{\xi_{n}^{2}+1}\right)
\end{gathered}
$$

Kinetic Overpotential

$$
\bar{\eta}_{p}^{k}=\frac{R T}{\alpha F} \ln \left(\xi_{p}+\sqrt{\xi_{p}^{2}+1}\right)
$$

Diffusion overpotential in the particles

$$
\begin{aligned}
& \bar{\eta}_{n}^{\text {diff }}=U_{n}\left(\theta_{n}^{s}\right)-U_{n}\left(\theta_{n}^{b}\right) \\
& \bar{\eta}_{p}^{\text {diff }}=U_{p}\left(\theta_{p}^{s}\right)-U_{p}\left(\theta_{p}^{b}\right)
\end{aligned}
$$

Mass transport overpotential in the electrolyte

Cell voltage

$$
\bar{\eta}_{e}^{m t}=\phi_{e}(L)-\phi_{e}(0)=\left(1-t_{+}\right) \frac{2 R T}{F} \ln \frac{c_{e}(L)}{c_{e}(0)}-\frac{I}{2 A}\left(\frac{\delta_{n}}{\kappa_{n}^{\text {eff }}}+2 \frac{\delta_{\text {sep }}}{\kappa_{\text {sep }}^{\text {eff }}}+\frac{\delta_{p}}{\kappa_{p}^{\text {eff }}}\right)
$$

$$
V(t)=U_{p}\left(\frac{c_{s, p}^{s}}{c_{s, p, \max }}\right)-U_{n}\left(\frac{c_{s, n}^{s}}{c_{s, n, \max }}\right)+\frac{2 R T}{F} \ln \left(\frac{\frac{-R_{s, p}}{6 \varepsilon_{s, p} i_{0, p} A \delta_{p}} I+\sqrt{\left(\frac{R_{s, p}}{6 \varepsilon_{s, p} i_{0, p} A \delta_{p}} I\right)^{2}+1}}{\frac{R_{s, n}}{6 \varepsilon_{s, n} i_{0, n} A \delta_{n}} I+\sqrt{\left(\frac{R_{s, n}}{6 \varepsilon_{s, n} i_{0, n} A \delta_{n}} I\right)^{2}+1}}\right)
$$

$$
+\left(1-t_{+}\right) \frac{2 R T}{F} \ln \frac{c_{e}(L)}{C_{e}(0)}-\frac{I}{2 A}\left(\frac{\delta_{n}}{\kappa_{n}^{e f f}}+2 \frac{\delta_{\text {sep }}}{\kappa_{\text {sep }}^{e f f}}+\frac{\delta_{p}}{\kappa_{p}^{e f f}}\right)
$$


Heat transfer and energy balance

Energy balance

$$
\frac{d}{d t} T=\frac{1}{M C_{p}}\left(\varphi_{\text {gen }}-\varphi_{\text {tra }}\right)
$$

Thermal flux generated during operation

Transferred thermal flux to environment

$$
\varphi_{\text {gen }}=-\left(\left(V-\left(U_{p}-U_{n}\right)\right) I+T \frac{d\left(U_{p}-U_{n}\right)}{d T} I\right)
$$

$$
\varphi_{\text {tra }}=h_{\text {conv }} A_{\text {cell }}\left(T-T_{a m b}\right)
$$

Internal temperature calculation

$$
T_{\text {int }}=T_{\text {skin }}\left(1+\frac{h_{\text {conv }} A_{\text {cell }}}{\lambda_{\text {cell }} r_{\text {cell }}}\right)-T_{\text {amb }} \frac{h_{\text {conv }} A_{\text {cell }}}{\lambda_{\text {cell }} r_{\text {cell }}}
$$

Coupling between 1D electrochemical and lumped thermal models

Arrhenius law applied to mass transport and kinetic parameters $\Psi$

$$
\Psi=\Psi_{r e f} \exp \left(\frac{E_{a}(\Psi)}{R}\left(\frac{1}{T_{r e f}}-\frac{1}{T}\right)\right)
$$

\section{Table III. Equations for the aging model. ${ }^{2}$}

SEI growth mechanism via solvent diffusion

Eq. Boundary conditions

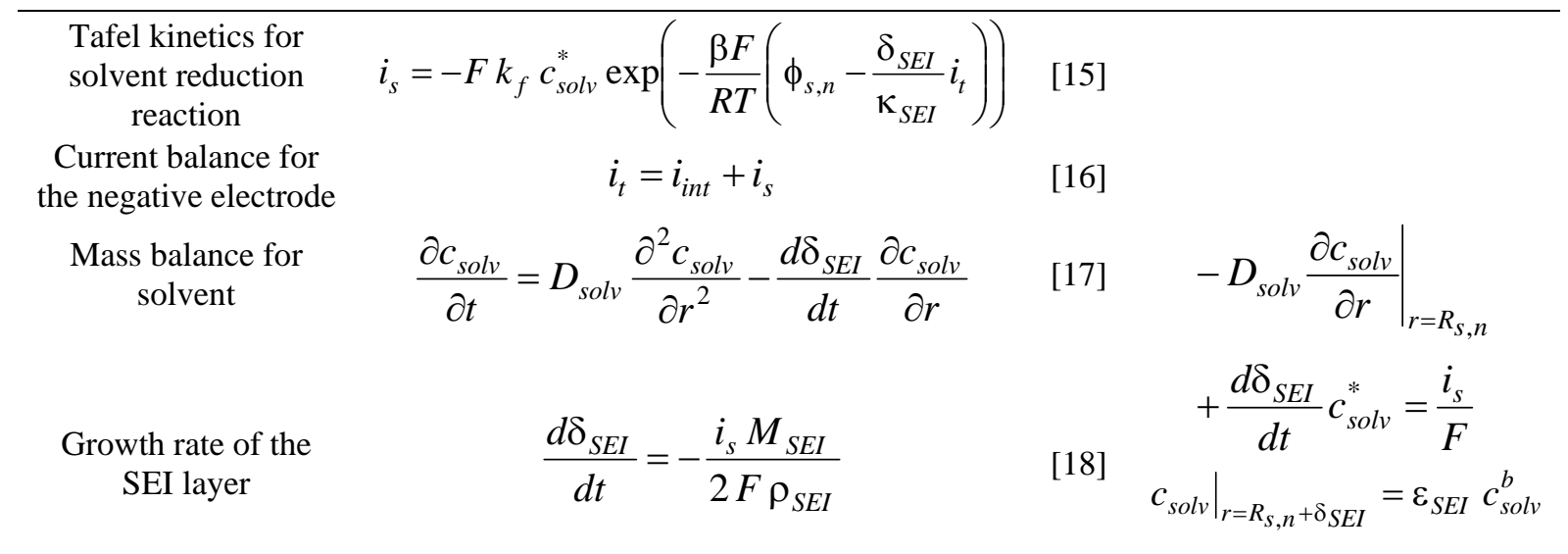


Table IV. Parameters of the electrochemical and thermal aging model.

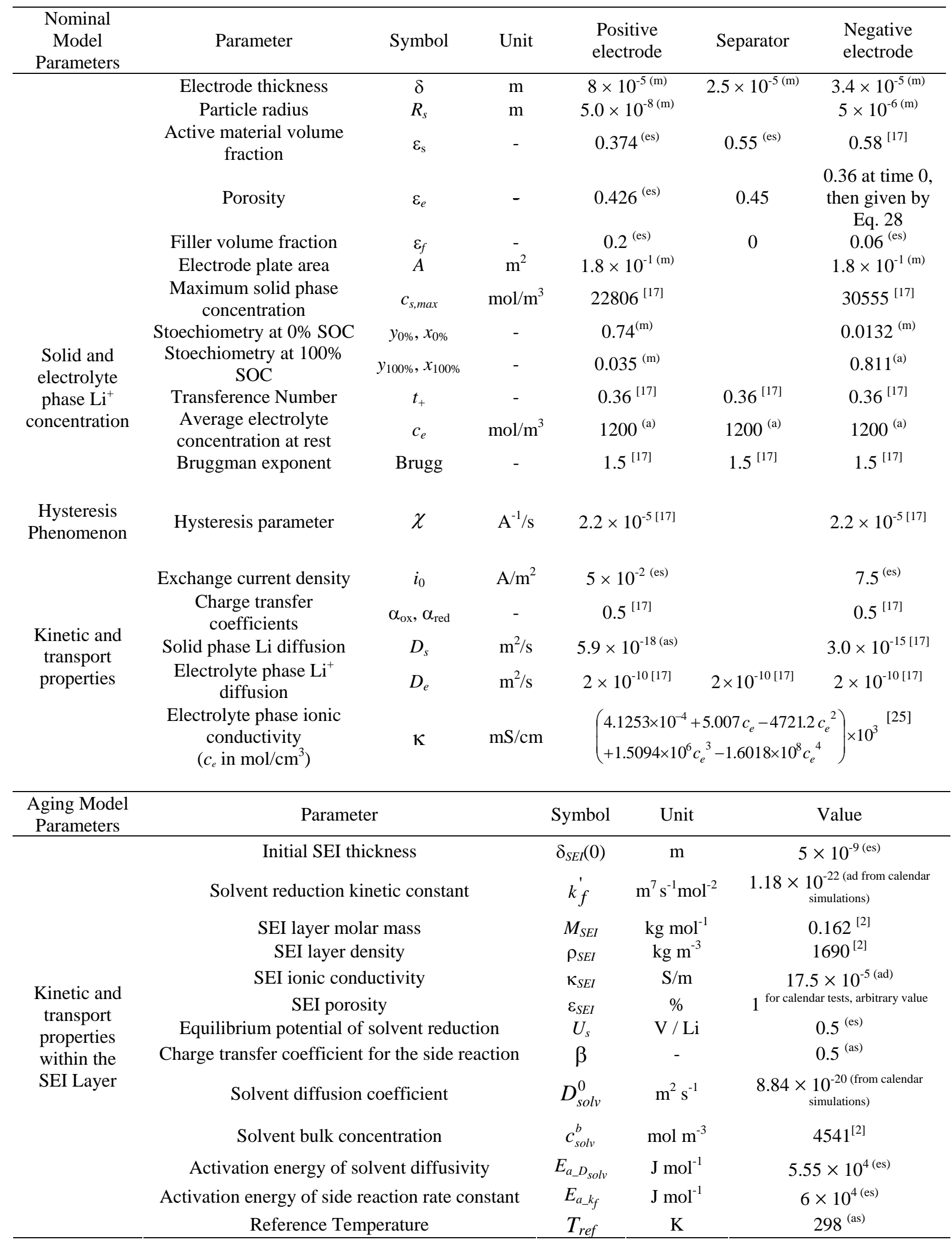

ad: adjusted, as : assumed without rationale, es: estimated, m: measured 


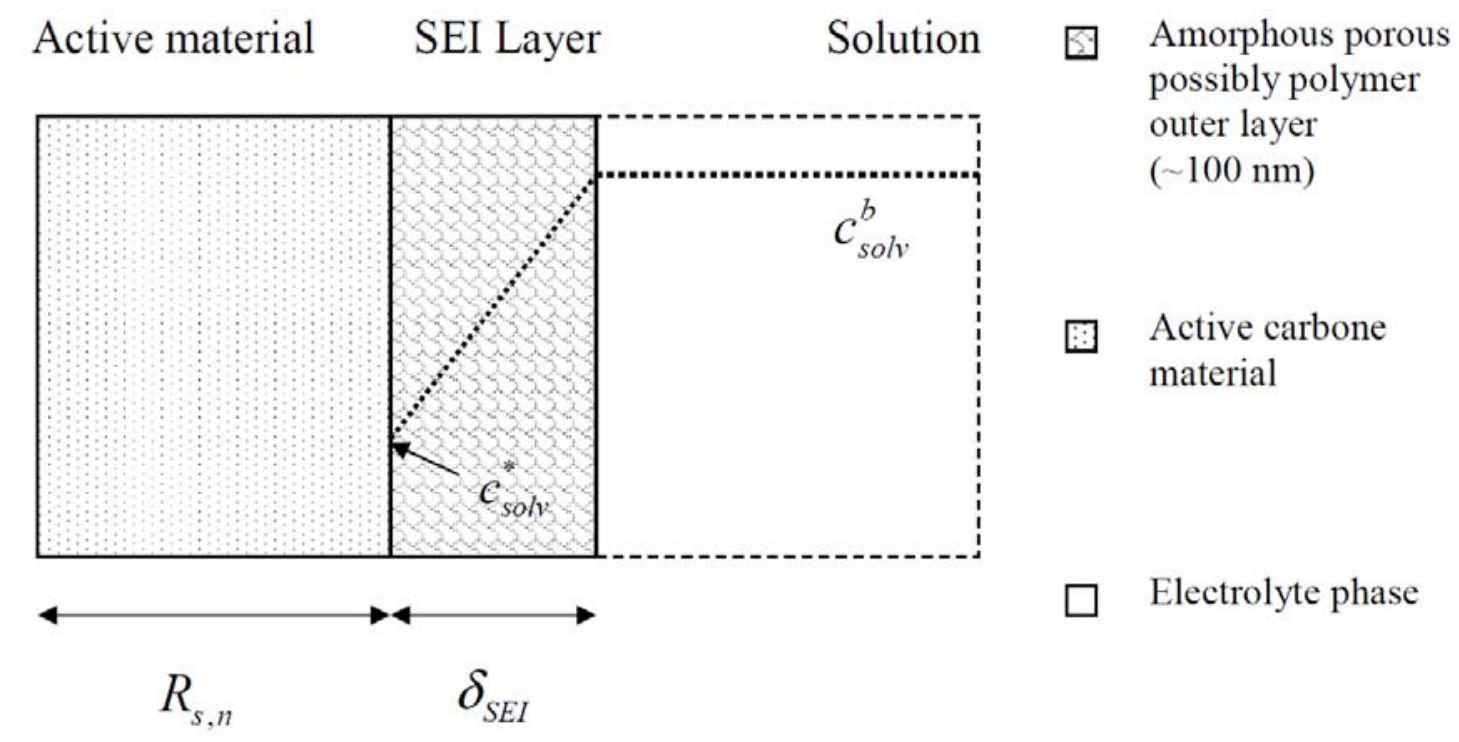

Figure 1. Schematic representation of the growing SEI film. 

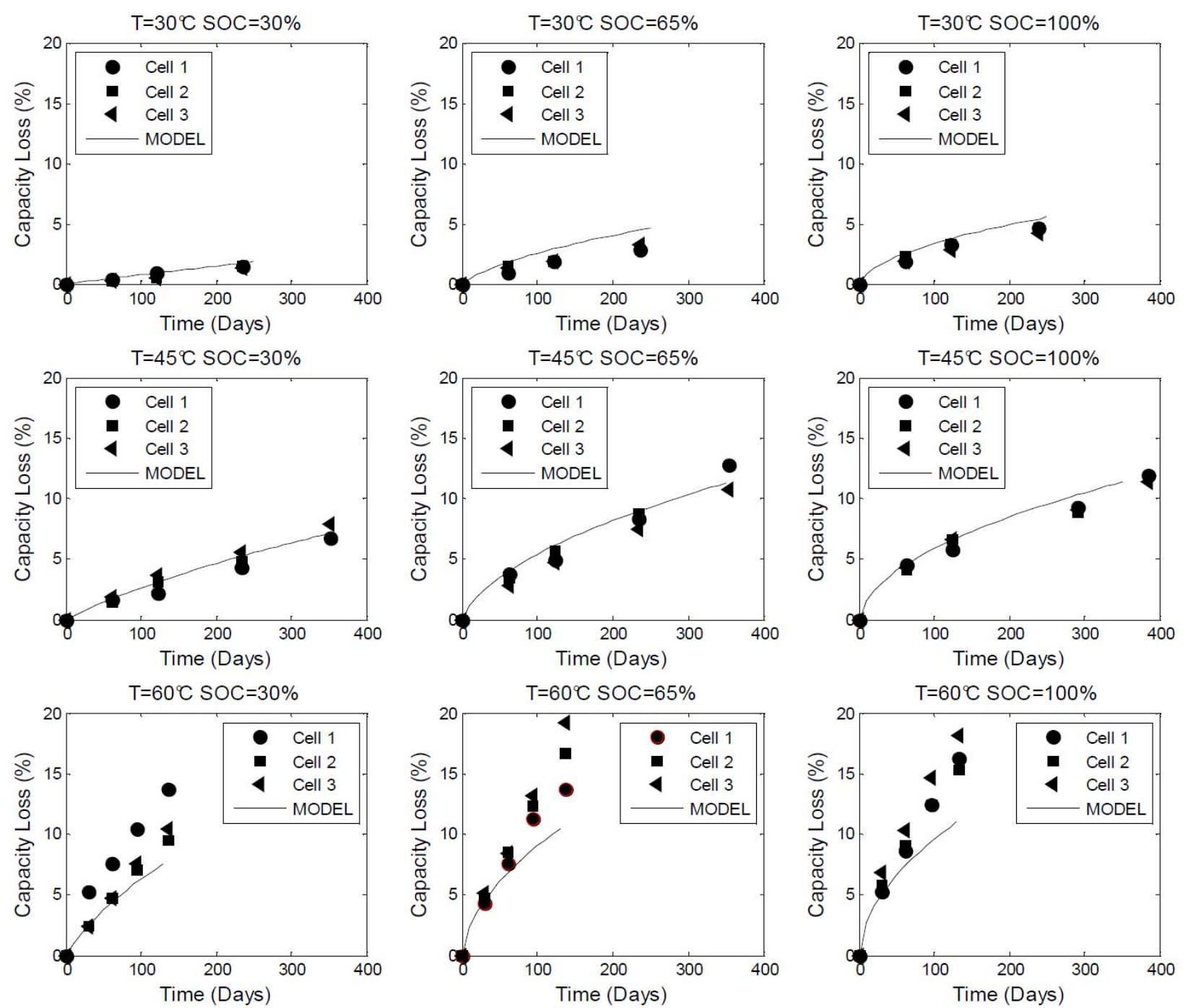

Figure 2. Model simulation of capacity loss compared to the experimental data of the calendar tests of the SIMCAL database. ${ }^{28}$ 

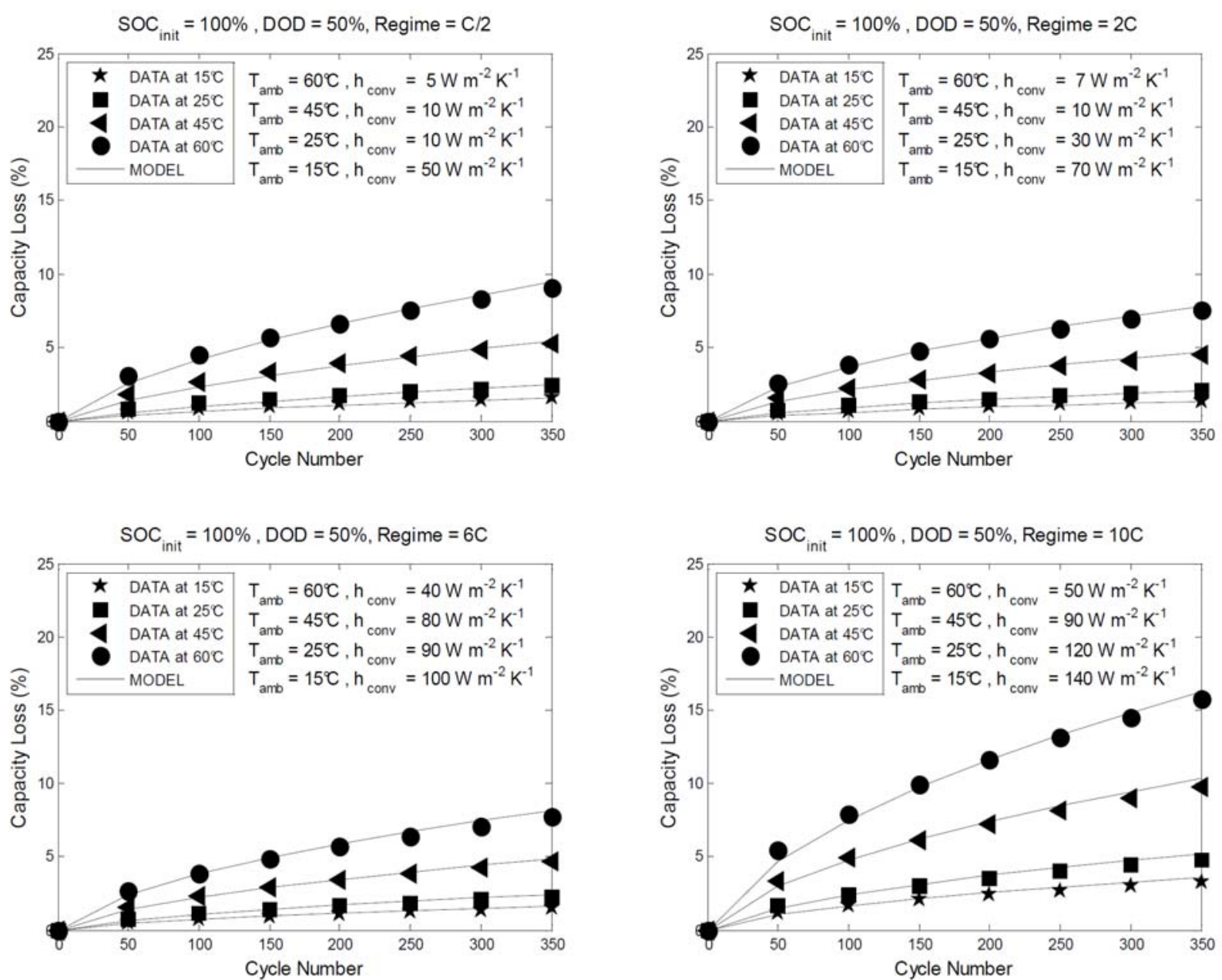

Figure 3. Model simulations of capacity loss compared to the modeled data of the cycling test database in Ref. 7. 


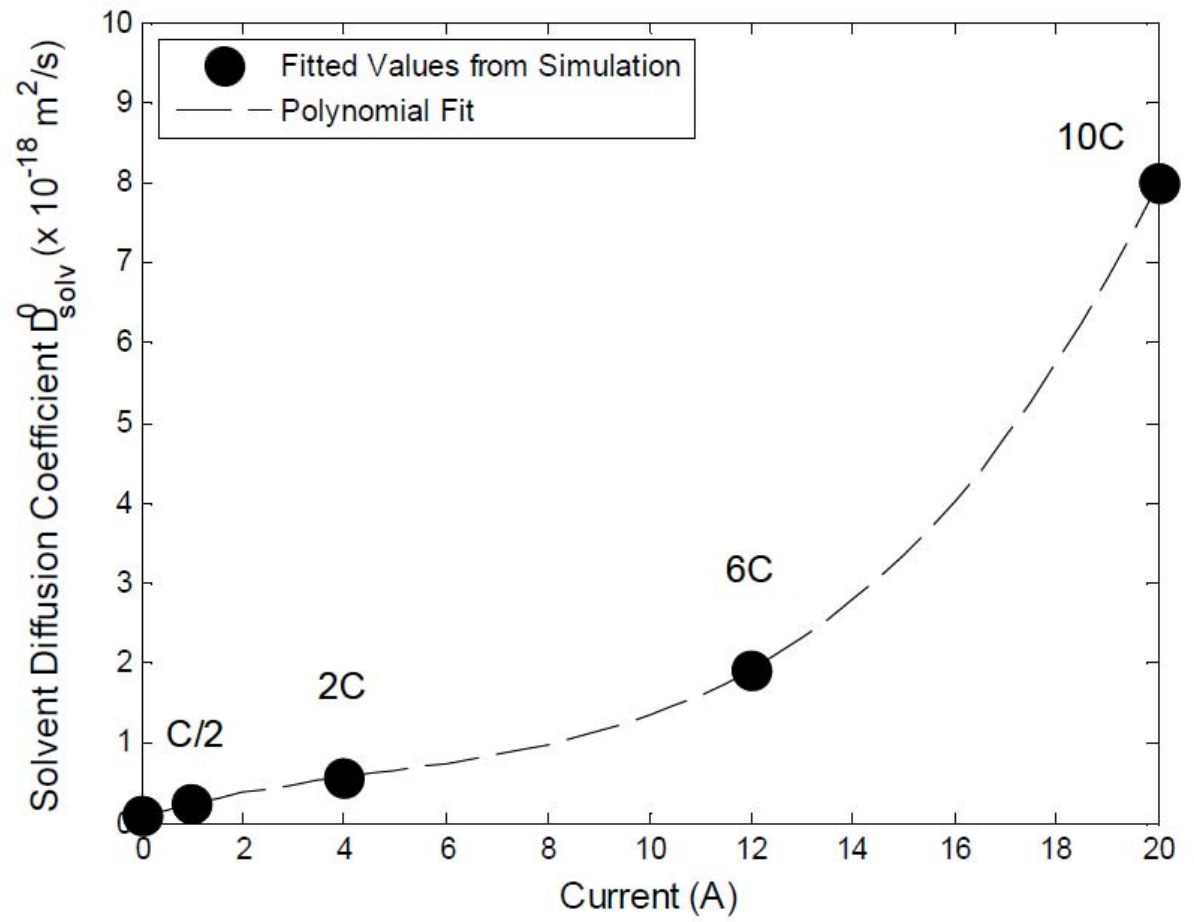

Figure 4. Evolution of the solvent diffusion coefficient as a function of current intensity. 


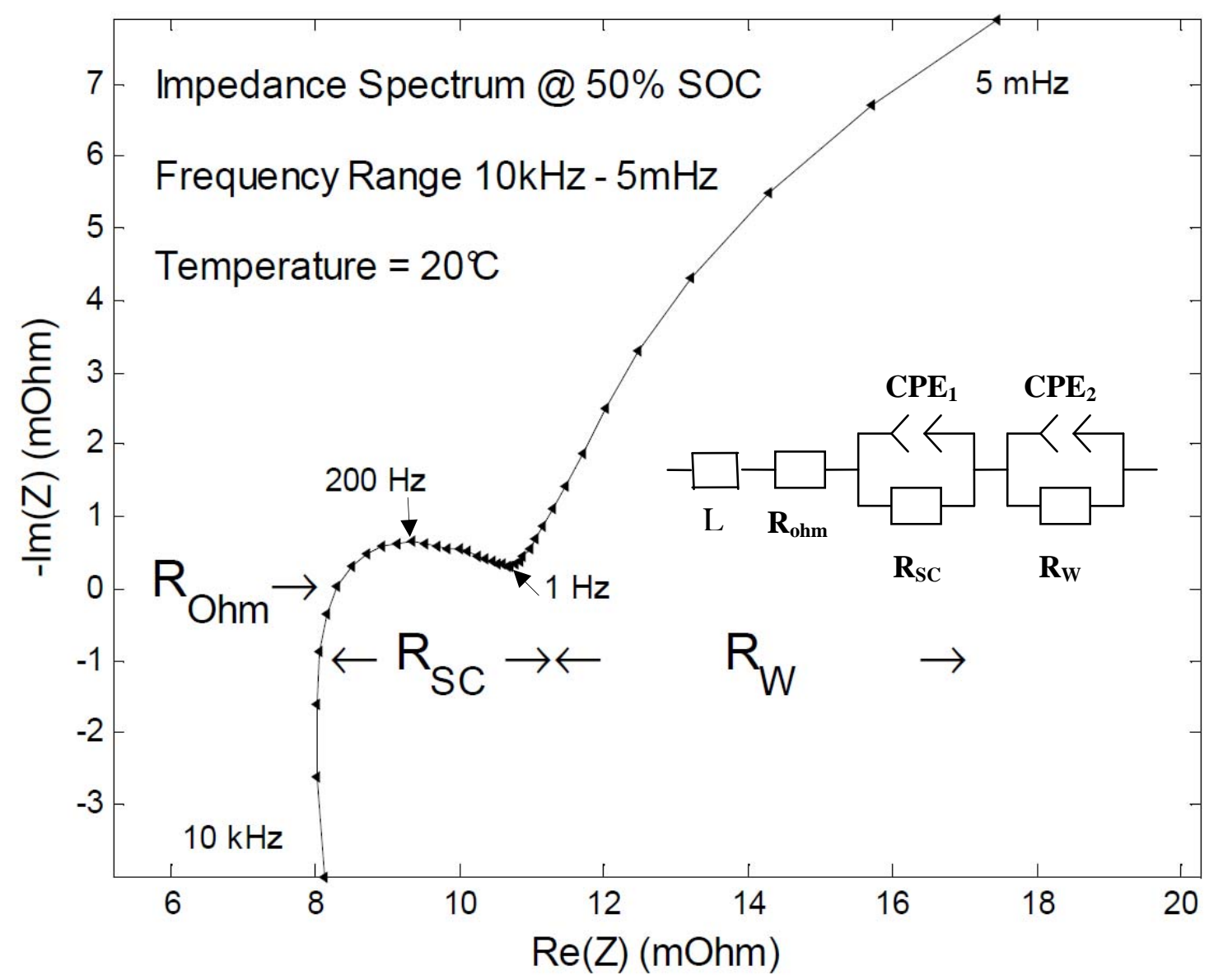

Figure 5. Impedance spectrum of the $\mathrm{LiFePO}_{4}$-graphite cell at $20^{\circ} \mathrm{C}$ and $50 \%$ SOC and equivalent circuit used to calculate $R_{o h m}$ and $R_{S C}$. 

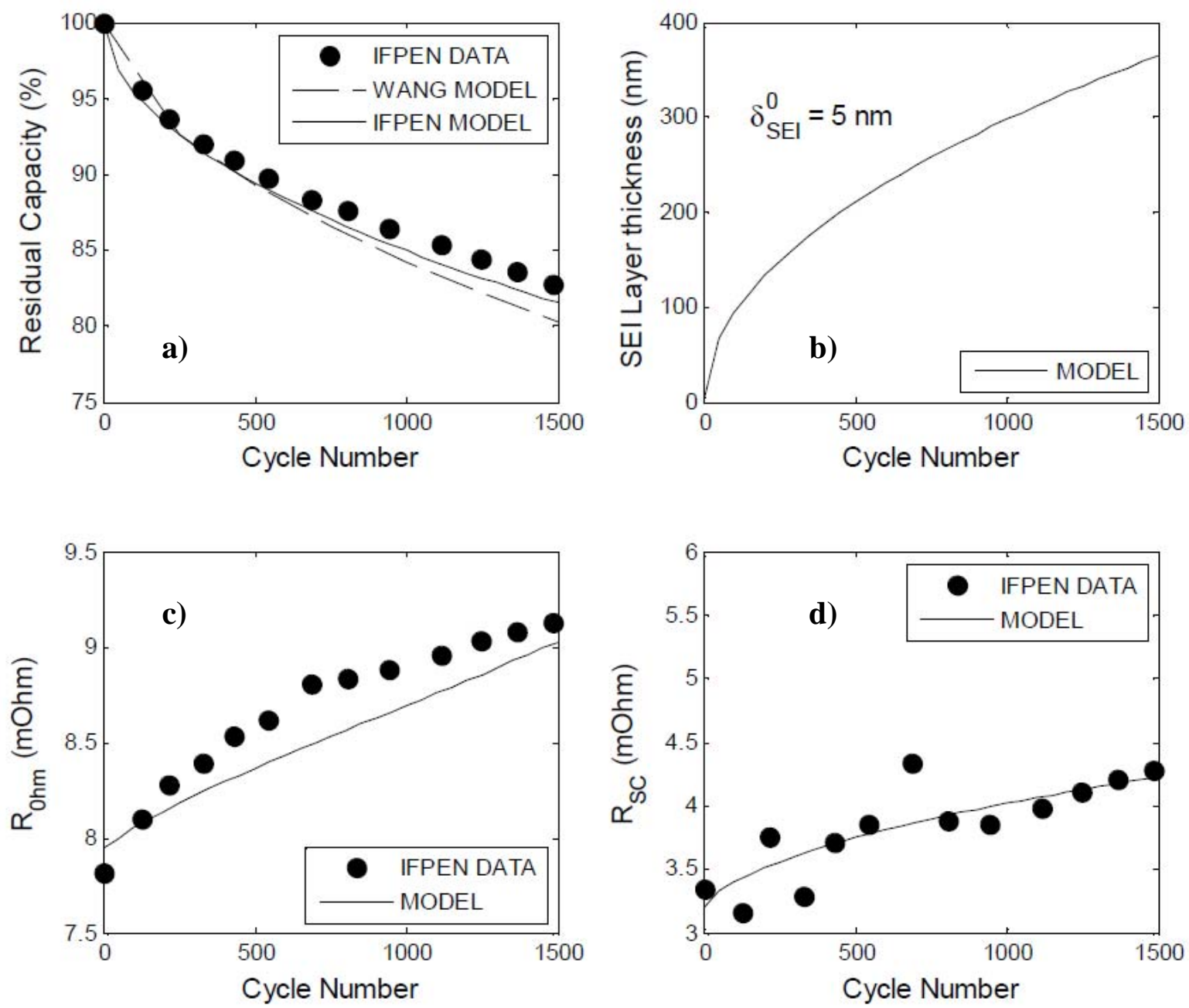

Figure 6. a) Residual capacity simulations compared to experimental data for C/2 cycling tests at $50 \%$ DOD from an initial SOC of $100 \%$ and $60^{\circ} \mathrm{C}$, b) Simulation of the SEI layer growth as a function of cycle number, c) Comparison between simulated and measured ohmic resistance as a function of cycle number, d) Comparison between simulated and measured semi-circle resistance as a function of cycle number. 

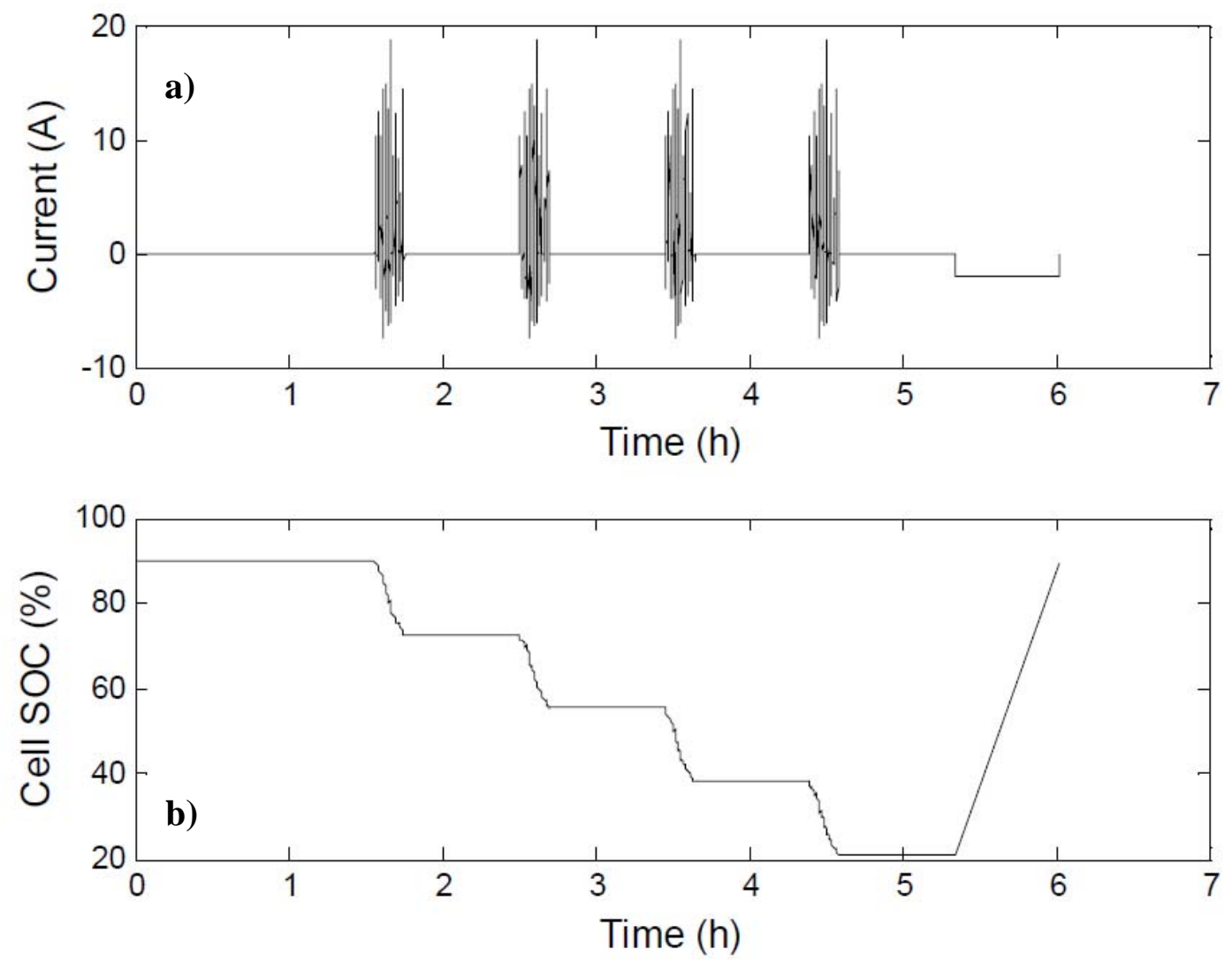

Figure 7. a) ZEV macro-cycle dynamic current profile as a function of time, b) evolution of the cell SOC during the macro-cycle. 

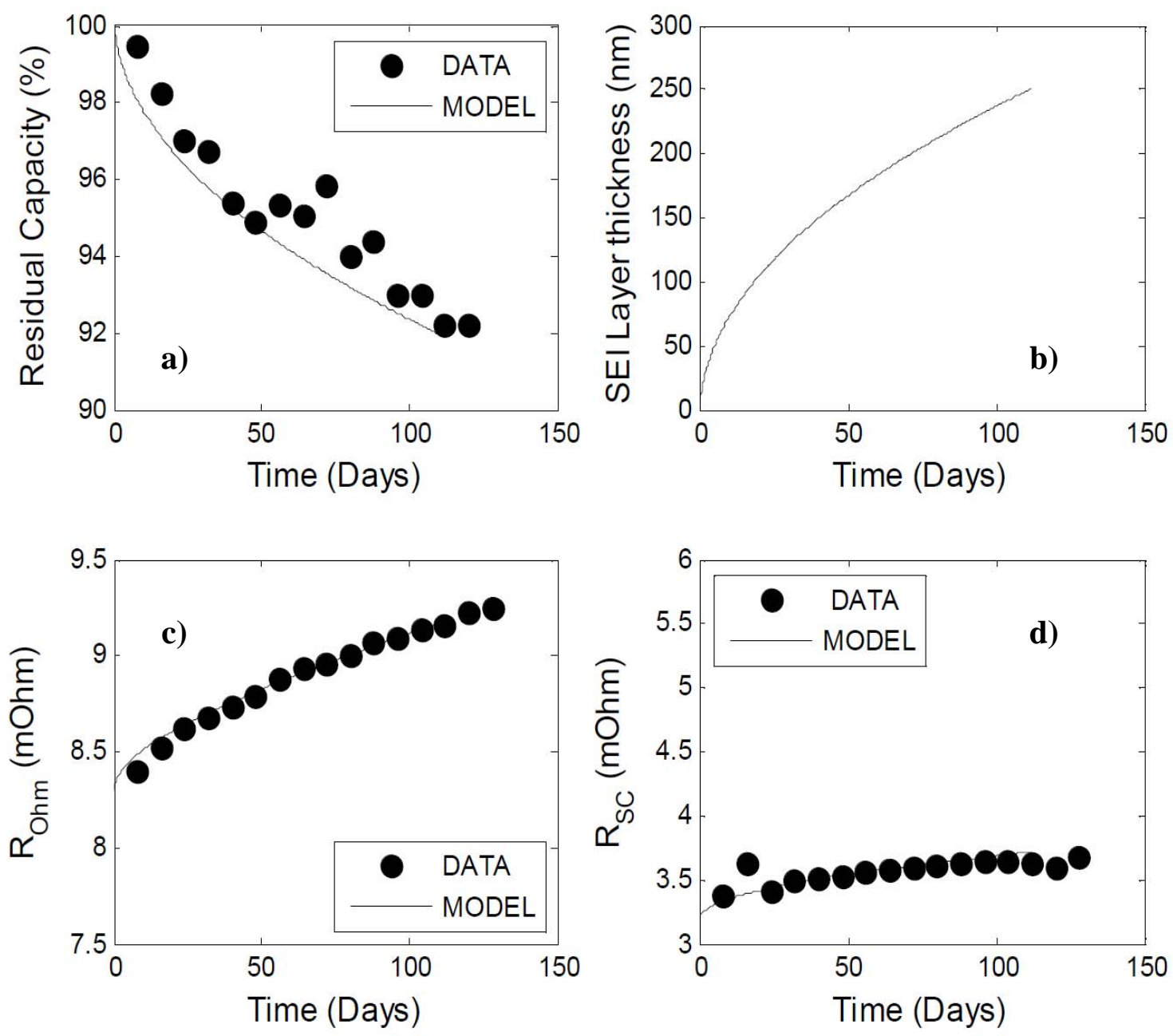

Figure 8. a) Residual capacity simulations compared to experimental data for the ZEV cycle test at $45^{\circ} \mathrm{C}$, b) Simulation of the SEI layer growth as a function of days, c) Comparison between simulated and measured ohmic resistance as a function of days, d) Comparison between simulated and measured semi-circle resistance as a function of days. 

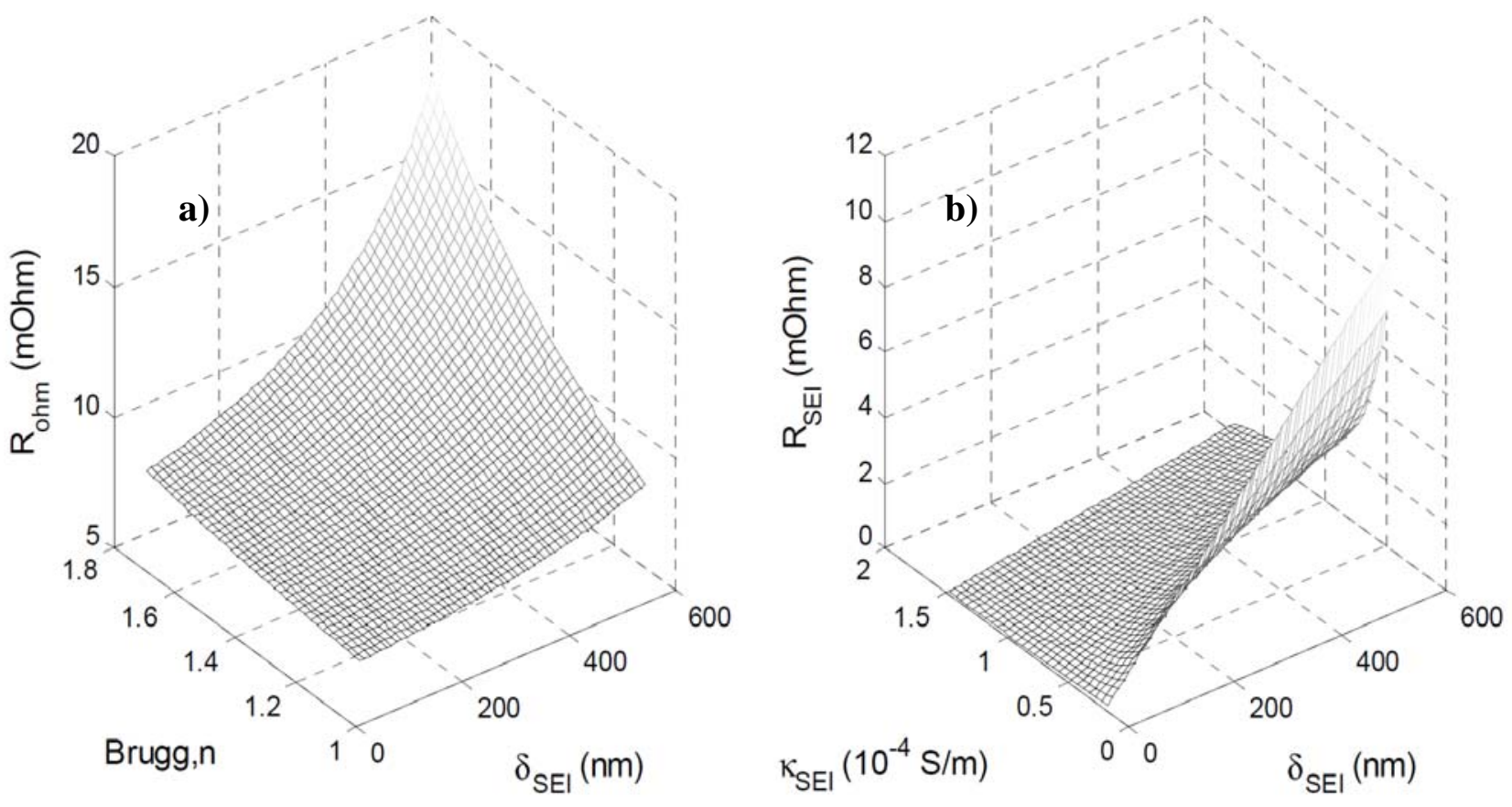

Figure 9. a) Evolution of the ohmic resistance as a function of the Bruggman exponent and the SEI film thickness, b) Evolution of the SEI resistance as a function of the film ionic conductivity and the film thickness. 